\title{
Eckart Hildebrandt \\ Betriebliche Personalpolitik als Spaltungsstrategie \\ des Kapitals - Kapitalistische Betriebspolitik und Gegenwehr in der Bundesrepublik 1974 - 1976
}

\section{Vorbemerkung}

Die folgende Untersuchung steht im Zusammenhang mit der weiter angelegten Analyse der Auswirkungen der Krise und der Arbeitslosigkeit in der Bundesrepublik auf die Lage und das Bewußtsein der Arbeiter, die in PROKLA 19/20/21 vom Redaktionskollektiv Gewerkschaften veröffentlicht wurde. Zum Zeitpunkt der Abfassung jener Untersuchung (Mitte 1975) konnten die Auswirkungen der Krise auf die Lage der Arbeiter erst sehr allgemein erfaßt und in ihren politischen Konsequenzen verfolgt werden. Es wurde gezeigt, wie die Krise und die sie begleitende Arbeitslosigkeit (deren struktureller Charakter auch von bürgerlichen Untersuchungen inzwischen nicht mehr verschleiert werden kann) die Konkurrenz unter den Arbeitern verschärft hat: Nicht nur die Konkurrenz zwischen unbeschäftigten und beschäftigten Arbeitern, sondern auch und gerade jene zwischen den beschäftigten Arbeitern selbst. Angesich ts des äußeren Drucks der industriellen Reservearmee vor den Betriebstoren und der innerbetrieblichen Rationalisierungsmaßnahmen in der Krise schälten sich die Differenzierungen innerhalb der Arbeiterschaft nach Qualifikation, sozialen und ethnischen Merkmalen deutlicher als zuvor heraus; ganze Arbeitergruppen wurden innerbetrieblich herabgestuft, Facharbeiter auf Arbeitsplätze gesetzt, die bisher angelernte Arbeiter innehatten, jene angelernten Arbeiter wurden entlassen. Gewachsene Kommunikationsstrukturen in und zwischen den Abteilungen wurden durch die Maßnahmen der betrieblichen Umstrukturierung zerschlagen; Dequalifizierungen, Intensivierung der Arbeit und Lohneinbußen mußten angesichts der verschärften Konkurrenz zwischen den Arbeitern hingenommen werden. Offenen Widerstand gab es dort, wo Massenentlassungen durchgesetzt wur* den (mit entsprechender Öffentlichkeitswirkung wie bei VW-Neckarsulm), oder dort, wo Betriebsräte und/oder Vertrauensleute in der Lage waren, diesen Widerstand in der Belegschaft trotz verschärfter Konkurrenz unter den Arbeitern zu organisieren.

Inzwischen stellt sich heraus, daß unsere damalige Analyse eher noch zu optimistisch formuliert wurde. Denn während sich Lernprozesse bei den Vertretern der Gewerkschaften erst in der anlaufenden Tarifrunde 1977 andeuten, haben die Unternehmen längst ihren Lernprozeß aus den veränderten Bedingungen gemacht: daß Massenentlassungen, eine verschleierte Informationspolitik über die Lage der Unternehmen und die zukünftige Personalpolitik, die rigide Durchsetzung einer ,,Herr im Haus"-Politik, zu geschlossenen und Solidarisierung fördernden Widerstandsaktionen führen können. Die veränderte Politik der Unternehmensleitung setzt heute nicht mehr auf spektakuläre Massenentlassungen, sondern auf die Konkurrenz innerhalb der Belegschaft, die Konkurrenz zwischen den Belegschaften der Einzelbetriebe eines Konzerns und auch die Konkurrenz zwischen nationalen Arbeiterklassen. Diesen Lernprozessen fortgeschrittener Unternehm enspolitik hinken die Gewerkschaften um einen ganzen Zyklus hinterher.

Das Gegeneinanderausspielen der verschiedenen Fraktionen der Belegschaften, das Hinüberziehen der durch das Betriebsverfassungsgesetz (BetrVG) eingebundenen Betriebsräte (BR) auf die Seite der durch Rentabilitätsüberlegungen geprägten Unternehmenspolitik, das Umgehen der derzeit durchgesetzten Schutzbestimmungen - kurz: das Scheitern der bisherigen gewerkschaftlichen Betriebspolitik an der neuen ökonomischen Situation und an den neuen Unternehmerstrategien sollen in der folgenden Untersuchung am Beispiel der Atuomobilindustrie dargestellt werden. 
Dabei ist - das sollte beachtet werden - die Automobilindustrie ein eher ,mildes ${ }^{\natural}$ Beispiel für die Unternehmenspohtik in der Krise, das gewählt wurde, weil hier die Materiallage (nicht zuletzt aufgrund der dort noch weitgehend funktionsfähigen betrieblichen Gruppen) relativ günstig ist. In anderen Branchen, wie etwa in der Textilindustrie, dürfte aufgrund des niedrigeren gewerkschaftlichen Organisationsgrades, der Zusammensetzung der Belegschaften und des großen Anteils von Klein- und Mittelbetrieben wesentlich rigider vorgegangen werden. Insofern sind die vorgelegten Thesen auch nur eingeschränkt verallgemeinerbar. Die folgende Analyse beschränkt sich in den ersten zwei Teilen im wesentlichen auf die Zusammenstellung des Materials, wobei der jeweiligen Unternehmenspolitik die Möglichkeiten und Grenzen der Politik der betrieblichen Interessenvertretung der Arbeiter entgegengestellt werden. Dabei wird deutlich, daß dieser Handlungsspielraum gegenüber der Unternehmenspolitik besonders durch die bisherige Betriebspolitik (einschließlich ihrer Determination durch das BetrVG) bestimmt ist, die in der Phase der Prosperität für die Belegschaft durchaus erfolgreich war, jetzt aber zum Hemmnis einer effektiveren Vertretung in der Krise wird. Ausgespart bleiben muß hier eine weitergehende Analyse der überbetrieblichen gewerkschaftlichen Politik und deren Möglichkeiten, bessere Rahmenbedingungen für die betriebliche Vertretung zu schaffen.

Während der erste Teil die Entlassungsstrategien in der Krise schildert, wird im zweiten Teil versucht, die Bedeutung der neueren Personalpolitik der Einzelkapitale als Spaltungsstrategie gegenüber der Belegschaft herauszuarbeiten. Im dritten Teil werden einige Schlußfolgerungen für die Formulierung einer Abwehrstrategie der Gewerkschaften und betrieblicher Gruppen gezogen, die sich hier aber noch ausschließlich auf die Betriebspolitik beziehen. Weitergehende Folgerungen müssen einer weiter gefaßten Diskussion überlassen bleiben.

\section{Teil 1}

\section{Betriebliche Maßnahmen der Anpassung der Belegschaften an das verringerte Produktionsvolumen in der Krise am Beispiel der Automobilindustrie}

Um Formen, Umfang und Bedeutung der Abbaumaßnahmen für die Situation der Beschäftigten und ihre Möglichkeiten der Gegenwehr angemessen beurteilen zu können, erweist es sich als notwendig, diese Maßnahmen differenzierter nach Zielsetzung und Auswirkungen darzustellen. Da nur eine repräsentative Untersuchung zu diesem Thema existiert (1) sowie eine beschränkte Zahl von Einzelinformationen aus schwer zugänglichen Quellen, kann ihr Umfang nur ungefähr an der Verringerung der Arbeitsplätze in der Industrie abgeschätzt werden.

Es werden nur die Maßnahmen betrachtet, die im Einzelbetrieb verhandelt und durchgesetzt werden. Der wichtige Bereich der Stillegungen und Teilstillegungen sowie der Unternehmenszusammenschlüsse muß hier ausgeklammert bleiben, obgleich gerade in solchen Fällen breitere betriebliche und überbetriebliche Gegenwehr stattfindet (Erwitt, Kalldorf, Speyer, Emden etc.). Im folgenden werden dabei den Maßnahmen der Unternehmen in der Regel die Gegenmaßnahmen der betrieblichen Interessenvertretung der Arbeiter gegenübergestellt.

1 Arbeitsberichte 4 und 5 des Projekts „Betriebliche und sozialpolitische Aspekte und Probleme betrieblicher Personalplanung", IFS Mïchen, 1975/76 
Nicht ausführlicher dargestellt sind Maßnahmen zur Arbeitsbeschaffung bzw. Arbeitsstreckung, um bei sinkender Produktion die Beschäftigten zu halten. Dazu gehört die Produktion auf Lager, das Vorziehen von Reparatur- und Erneuerungsarbeiten, die Ausweitung von Aus- und Fortbildungsmaßnahmen, die Rücknahme von Fremdaufträgen und schließlich Veränderung des Sortiments und die Versuche, neue Absatzmöglichkeiten zu erschließen. Um Entlassungen abzuwehren, fordern viele BR gerade solche Maßnahmen und beteiligen sich teilweise an Initiativen zur Absatzsicherung. Abgesehen davon, daß solche Maßnahmen nur geringe Beschäftigungswirkung haben geraten sie dabei häufig in den Widerspruch zwischen betrieblicher Arbeitsplatzsicherung und gesellschaftspolitischen Gewerkschaftszielen (2). Bei der Stornierung bzw. dem Hereinholen von Fremdaufträgen wird unmittelbar deutlich, wie die Konkurrenz zwischen Einzelunternehmen direkt auf die BR-Politik durchschlagen kann.

\section{Quantitativer Umfang der Verringerung der Beschäftigung in der Krise}

Zunächst eine kurze Gesamtübersicht:

Die erste Tabelle zeigt die Beschäftigungsentwicklung insgesamt in der Industrie und in ausgewählten Industriegruppen. Dabei zeigt sich, daß der Beschäftigungsabbau im Straßenfahrzeugbau noch unterhalb des Industriedurchschnittes liegt:

Die zweite Tabelle zeigt den sehr unterschiedlichen Beschäftigungsabbau der verschiedenen Belegschaftsgruppen im Verarbeitenden Gewerbe:

Die dritte Tabelle zeigt die Belegschaftsveränderungen in den einzelnen Automobilkonzernen und den Umfang der Arbeitszeitflexibilisierung durch Kurzarbeit/ Sonderschichten.

2 Markante Beispiele hierfür sind:

Die Stellungnahme der BR von 9 Rüstungsunternehmen vom 27.10.75, in der gefordert wird: ,,Wenn der Bund vorübergehend die Kapazitäten nicht auslasten kann, sollte der Bund aus seiner Fürsorgepflicht heraus die Hereinnahme von Füllaufträgen aus Nichtspannungsgebieten zuzulassen, wobei der Begriff, Nichtspannungsgebiete ${ }^{6}$ nicht melr so eng ausgelegt werden dürte wie in der Vergangenheit."

Der BR-Vorsitzende von Opel-Bochum forderte in der Westfälischen Rundschau vom 12.2.74 von der Bundesregierung die Aufhebung der Geschwindigkeitsbegrenzung, da sie die Autokäufer verunsichere. Der Opel-Gesamtbetriebsrat schrieb in diesem Sinne an den Bundeskanzler (vgl. Express $1 / 75$, S. 6)

Die ÖTV und die BR der Nordwestdeutschen Kraftwerke AG riefen gegen die Besetzung des Bauplatzes des Kernkraftwerkes Brokdorf/Unterelbe zu einer Gegendemonstration zur Sicherung der bestehenden und zur Schaffung neuer Arbeitsplätze auf. Die Absage der Gegendemonstration begründete die ÖTV damit, daß es nicht zu verantworten sei, , wenn Belegschaftsmitglieder von Chaoten zusammengeschiagen würden". Gemeint war die "Bürgerinitiative Umweltschutz Unterelbe": Vgl. FR vom 1.11.76 
Tabelle I: Beschäftigte in ausgewählten Industriegruppen

\begin{tabular}{|c|c|c|c|c|c|c|c|}
\hline & \multicolumn{6}{|c|}{ Jahresdurchschnitte in 1.000} & \multirow{2}{*}{$\begin{array}{l}\text { Veränderung (\%) } \\
\text { Okt, } 73 \text { gegenüber } \\
\text { Tiefststand } 75 / 76\end{array}$} \\
\hline & 1970 & 1971 & 1972 & 1973 & 1974 & 1975 & \\
\hline Gesamte Industrie & 8603,4 & 8537,9 & 8340,2 & 8368,4 & 8143,5 & 7615,9 & $-14,7$ \\
\hline Kohlenbergbau & 277 & 274 & 252 & 232 & 224 & & \\
\hline Steine und Erden & 231 & 237 & 241,5 & 238,7 & 219,4 & 19 & $-27,3$ \\
\hline Eisenschaffende Ind. & 334,6 & 326 & 311,9 & 315,7 & 316,0 & 310 & $-\quad 5,7$ \\
\hline NE-Metall Ind. & 91,6 & 89,6 & 87,1 & 90,6 & 90,0 & 82 & $-14,0$ \\
\hline Maschinenbau & 1119,8 & 1117,5 & 1083,3 & 1086,4 & 1089,2 & 1040,2 & $-9,2$ \\
\hline Straßenfahrzeugbau & 606,2 & 630,9 & 609,2 & 625,8 & 611,0 & 566,7 & $-11,7$ \\
\hline Schiffbau & 77,9 & 77,6 & 75,3 & 72,0 & 73,1 & 76,2 & $+0,8$ \\
\hline Elektrotechn. Ind. & 1094,5 & 1066,7 & 1051,5 & 1089,3 & 1089,8 & 999,2 & $-13,7$ \\
\hline Themische Ind. & 595,7 & 589,0 & 580,3 & 587,3 & 600,5 & 582,6 & $-4,1$ \\
\hline Textilindustrie & 501,5 & 481,5 & 458,1 & 434,0 & 393,8 & 356,9 & $-20,0$ \\
\hline Bekleidungsind. & 379,1 & 371,6 & 372,2 & 359,7 & 310,2 & 288,3 & $-21,9$ \\
\hline
\end{tabular}

Quelle: Wirtschaft und Statistik, Heft 2/75, S. 84; Heft 5/76, S. 290; Heft 9/76, S. 573 und eigene Berechnungen

Tabelle II: Verringerung der Beschäftigtenzahlen im verarbeitenden Gewerbe (in 1.000)

Abhängig Veränderg. Ange- Arbeiter Verändg. Frauen Verändg. Ausländer Verändg. Beschäft. $\%$ stellte $\%$ \%

$\begin{array}{llllllllll}1970 & 9.828 & -0,46 & 2.580 & 7.247 & -1,62 & 2.935 & -1,43 & \\ 1971 & 9.782 & -1,63 & 2.651 & 7.129 & -1,79 & 2.893 & -1,10 & \\ 1972 & 9.622 & +0,60 & 2.619 & 7.001 & +0,52 & 2.861 & +1,71 & 1.376 \\ 1973 & 9.680 & -2,36 & 2.640 & 7.038 & -3,32 & 2.910 & -2,78 & \\ 1974 & 9.451 & -5,76 & 2.645 & 6.804 & -8,09 & 2.829 & -6,92 & 1.416 \\ 1975 & 8.906 & & 2.650 & 6.253 & & 2.633 & & 1.219\end{array}$

$1975 / 73$
(\%) $\quad-7,99$
$+0,38-11,01$
$-9,52$

Daten und eigene Berechnungen nach Arbeits- und sozialstatistik 10/76, S. 312. Vollständige Zahlenreihen zu den ausländischen Arbeitern sind wegen einer Umstellung der Statistik nicht erhältlich.

Tabelle III:

Mitarbeiter im Inland
am Jahresende
$73 \quad 74$

$\begin{array}{lrr}\text { Audi NSU } & 33.8 & \\ \text { Audi NSU } & 33.876 & 28.936 \\ \text { BMW } & 27.737 & 25.805 \\ \text { Daimler-Benz(1) } & 126.855 & 122.899 \\ \text { Ford } & 52.702 & 48.941 \\ \text { Opel } & 57.364 & 46.200 \\ \text { Porsche } & 4.335 & 3.461 \\ \text { VW } & 125.787 & 111.527\end{array}$

Niedrigststand Produktionskürzg. Anz.d. i. dieser Periode (Kurzarb) i. Mio. Son(Zeitpunkt)

$$
73 \begin{array}{ccc}
\text { Stunden(2) } & \text { der- } \\
74 & 75 & \text { schi. }
\end{array}
$$

1. Inlands-Ko nzern, einschl. Nutzfahrzeugwerke; 2. Ermittelt aufder Basis von 1 -werten: Inden Fällen Porsche $u$. VW eigene Berechnungen m. Hilfev. Firmenangaben. 3. Keinerlei Kurzarb. i.d.PkwProd.; vergleichbare Angaben ï. gelegentl. Kurzarb. i.d. Spalte „leichte Nutzfahrzeuge" liegen nicht vor. 4. Keine verwertbaren Angaben; 5. 2 Sonderschichten $=1$ Tagesprod ; 6. Partielle Überzeitarbeit im Aggregatebau.

(Quelle: Die Zeit vom 23.1.76) 
2. Maßnahmen zur Verringenung des Arbeitsvolumens durch Verringerung der Arbeitszeit:

\subsection{Verlängerung des Werksurlaubs, unbezahlter Urlaub:}

Diese Maßnahmen wurden nur sehr beschränkt und dann bei ausländischen Arbeitern angewandt. Für diese dann das Risiko des Arbeitsplatzverlustes (vgl. Anlaß des Streiks bei Ford/Köln 1973).

Relativ häufig wurde ein verlängerter bzw. zusätzlicher Betriebsurlaub mit $\mathrm{Zu-}$ stimmung des BR's ( 87 BetrVG) durchgeführt

\subsection{Abbau von Mehrarbeit:}

In der Mehrzahl der untersuchten Unternehmen wurde zu Beginn der Krise Mehrarbeit geleistet. Sonderschichten wurden sehr früh abgebaut (Frühjahr 1973), sie zeigen früh einen Konjunkturumschwung an. Überstunden scheinen weniger konjunkturempfindlich, sie wurden auch im Tiefpunkt der Krise nie ganz abgebaut. In der KfZ-Industrie werden Überstunden hauptsächlich von Zeitlöhnern, die in Reperatur-, Instandhaltungs- und Einrichtertätigkeiten beschäftigt sind geleistet. Der dort gegebene ungleichmäßige Arbeitsanfall findet auch in der Krise statt (z. B. Modellwechsel) und kann aus Qualifikationsgrïnden kaum von Beschäftigten aus anderen Abteilungen, die von Entlassung bedroht sind, geleistet werden. Im Angestelltensektor wurden die Überstunden unterproportional eingeschränkt.

Die Einschränkung von Überstunden war zwar die am häufigsten eingesetzte Maßnahme (66\% der in allen Branchen befragten Betriebe), sie ist sehr schnell realisierbar, hat aber nur ein relativ geringes Arbeitsschaffungspotential (die Stu die schätzt $\max$. ca. $3 \%$ ).

Für das Unternehmen stellt sich der Abbau von Mehrarbeit sehr günstig, da die Lohnkosten aufgrund der Zuschläge überproportional verringert werden und der BR über keine Einspruchsrechte beim Abbau verfügt.

Die BR's befinden sich in einer widersprüchlichen Situation: Sonderschichten und Überstunden haben in den letzten Jahren in der PKW-Produktion ihren Ausnahmecharakter verloren; die entsprechenden, beträchtlichen Zusatzeinkommen werden inzwischen als normal angesehen. Andererseits streben sie die Einhaltung der tariflich geregelten Arbeitszeit an. Für sie gilt es also, durch eine Verringerung des Arbeitsvolumens/Beschäftigter Entlassungen zu vermeiden, andererseits die Einkommenseinbußen möglichst gering zu halten (sie betrugen bis zu $20 \%$ !). Ein drittes Problem liegt in der ungleichen Verteilung von Sonderschichten/Ubberstunden auf die Belegschaft und der entsprechend ungleichen Wirkungen ihres Abbaus. So insbesondere, wenn in verschiedenen Abteilungen eines Betriebs gleichzeitig Unterbeschäftigung in einigen, Mehrarbeit in anderen besteht. Nur in Einzelfällen konnte dies durch Umsetzungen innerhalb des Betriebes/Unternehmens ausgeglichen werden (in einem Fall wurden 1000 Ausländer in ein mehrere hundert Kilometer entferntes Zweigwerk versetzt).

Generell aber waren kurzfristige Umsetzungen kaum möglich. Auch konnten sich die BR gegen die Einführung von Überstunden in einigen Abteilungen bei gleich- 
zeitigem Beschäftigungsabbau kaum wehren (Mitbestimmungsrecht nach $\S 87$, Abs. 1.3 BetrVG), da im Weigerungsfalle die Einigungsstelle ( $\$ 87$, Abs. 2) den unternehmerischen Begründungen zuneigen wird (3) (z. B. Überstunden des Einrichtepersonals bei Modellwechsel, um die Marktposition zu verbessern).

\subsection{Kurzarbeit:}

Diese Maßnahme wurde im Verarbeitenden Gewerbe und im Baugewerbe sehr häufig durchgeführt. Mehrheitlich in Großbetrieben. Betroffen waren weitgehend Produktionsarbeiter.

Entlassungen ausgenommen, ist diese Maßnahme am beschäftigungswirksamsten. Sie wurde normalerweise nach der Verringerung von Überstunden/Sonderschichten und vor Entlassungen durchgeführt. Die Unternehmen bevorzugen diese Maßnahme, da sie schnell einsetzbar, sehr wirksam und aufgrund der Lohnersatzleistungen der Bundesanstalt für Arbeit sehr kostengünstig ist. Der Hauptvorteil gegenüber anderen Maßnahmen besteht für die Unternehmer darin, daß durch die Flexibilisierung des betrieblichen Arbeitskräftepotentials entscheidende Weichenstellungen bei gleichzeitiger Verringerung der Lohnkosten aufgeschoben werden können, die sich bei einer Änderung der Marktkonstellation als verhängnisvoll erweisen könnten, und die Anpassung später und damit unter sichereren Einschätzungen erfolgen kann.

Einschränkungen in der Anwendung dieses Instruments liegen in den betrieblichen Voraussetzungen der Gewährung ( $\S 64 \mathrm{AFG}$ ), die Begrenzung der Bezugszeit ( $\$ 67$ AFG), dem Mitbestimmungsrecht des Betriebsrates ( $\$ 87$ Abs. 1 Satz 3 BetrVG) sowie der Erfahrung, daß bei längerer Kurzarbeit eine Tendenz zur Abwanderung der Facharbeiter eintritt.

Anspruchsvoraussetzungen für die Gewährung von Kurzarbeitergeld sind:

a. ein Arbeitsausfall aus wirtschaftlichen Ursachen einschließlich betrieblicher Strukturveränderungen und unabwendbarer (Natur-)Ereignisse;

b. dessen Unvermeidbarkeit;

c. ein zeitlich beschränkter Arbeitsausfall (grundsätzlich bis zu 6 Monaten) bei einem Mindestbelegschaftsteil (mindestens ein Drittel der im Betrieb bzw. Abteilung Beschäftigten) und einem Mindestumfang des Arbeitsausfalls (10\% der Arbeitszeit). Damit hoffte der Gesetzgeber Tatbestände umschrieben zu haben, die sicherstellen, „daß durch die Gewährung von Kurzarbeitergeld den Arbeitnehmern die Arbeitsplätze und dem Betrieb die eingearbeiteten Arbeitnehmer erhalten werden." ( $\$ 63$ Abs. 1 AFG). Dazu wird dem Betrieb zusätzlich auferlegt, durch alle erdenklichen Maßnahmen die Kurzarbeit zu verhindern oder aber zu verringern (Unvermeidbarkeit) (4).

3 Vgl. hier z. B. die Auseinandersetzung um die Genehmigung von Sonderschichten bei Opel/Bochum

4 Vgl. Karl W. Kühn, Kurzarbeit-Fibel, Stuttgart 1976, S. 46

Solche Maßnahmen können sein:

- Arbeit auf Lager;

- Umsetzung der Kurzarbeiter in andere, voll arbeitende Betriebsabteilungen; 
Die derzeitigen gesetzlichen und tariflichen Bestimmungen reichen keinesfalls aus, um Mißstände auszuschließen und zu verhindern, daß Kurzarbeit zur Vorbereitung von Entlassungen dient. Dabei ist immer davon auszugehen, daß das Kurzarbeitergeld aus dem Fond der Arbeitslosenversicherung gezahlt wird, also von den Belegschaften selbst aufgebracht wird.

$\mathrm{Zu}$ den Mißständen gehört die Konzentration von Kurzarbeitsphasen auf Zeiträume mit hohem Feiertagsanfall, auf Zeiträume, in denen technisch bedingte Produktionspausen notwendig sind sowie die Gleichzeitigkeit von Kurzarbeit und Mehrarbeit. „Besonderns auffälig verhielten sich einige Automobilunternehmen, die, in Kenntnis einer verbesserten Auftragslage, Kurzarbeit einlegten, bei der Bundesanstalt in Nürnberg abkassierten und Wochen später froh Sonderschichten verordneten. Seit geraumer Zeit erhärtet sich ferner bei der Bundesanstalt der Verdacht, daß Unternehmen, die technisch bedingte Produktionspausen einlegen müssen, Kurzarbeit anmelden, um so Lohnkosten zu sparen. Besonders auffällig ist jedoch die Tatsache, daß vor den Weihnachts- und Osterfeiertagen eine verstärkte Tendenz zur Kurzarbeit festzustellen ist ... Bei einem großen Chemiekonzern, der in jünster Zeit mit Schlagzeilen über Kurzarbeit von sich reden machte, schaffen an normalen Tagen die Beschäftigten zum Teil bis zu 12 Stunden. “ (5)

Die Regelungen schützen die Belegschaften auch nicht vor den Versuchen der Unternehmen, die Restarbeitszeit zu intensivieren und neben der Lohnkosteneinsparung noch Produktivitätserhöhungen durchzusetzen. Dies ist unter dem Druck drohender Entlassungen besonders leicht möglich. „Die Pelikan-Werke in Hannover verordneten Kurzarbeit, um Lager abzubauen. Der unerwartete Effekt: es wurde noch mehr produziert. Die kurzen Erholungspausen wurden nicht mehr genutzt, die vorgeschriebenen Arbeitszeiten wurden streng eingehalten, der Krankenstand ging zuruick." (6)

Eine weitere Zweckentfremdung ist im Gesetz selbst angelegt, indem zu den betrieblichen Voraussetzungen auch ,betriebliche Strukturveränderungen“ gezählt werden ( $\$ 64$ Abs. Satz 1 AFG) (7).

Diese Regelung ermögicht es den Unternehmen, Kurzarbeitsphasen zur Über-

- Durchführung von Aufräumungs-, Instandsetzungs- und Füllarbeiten;

- Urlaubsgewährung;

- Zumutbare Entlassung eines Teils der Belegschaft.

So kann die Arbeitsverwaltung von einem Unternehmen vor Gewährung des Kurzarbeitergeldes die Entlassung der beschäftigten Nicht-EG-Ausländer verlangen vgl. Arbeitsbericht 4 des IFS München, S. 67.

Die Vermeidbarkeit des Arbeitsausfalls wird ferner angenommen, wenn unmittelbar vor Einfuhrung der Kurzabeit in nennenwertem Umfang Überstunden geleistet wurden.

$5 \quad$ Welt der Arbeit v. 31.10.75

6 Die ZEIT v. 28.2.75, zitiert nach Arbeitsblätter für Teilnehmer zum Themenkreis „Betrieb", 1. Thema, S. 5, hg. v. DGB-Bundesvorstand

7 Merkblatt der BA zum Kurzarbeitergeld: „Eine betriebliche Strukturveränderung kann sowohl durch die Umstellung auf ein neues Produkt, durch Erweiterung oder Einschränkung der Fertigung als auch durch innerbetriebliche Umorganisation, z. B. Automation bewirkt werden. Eine betriebliche Strukturveränderung in diesem Sinne liegt nur vor, wenn sie durch die allgemeine wirtschaftliche Entwicklung bedingt ist." 
holung, Umgruppierung und Erneuerung des Maschinenparks zu nutzen und Rationalisierungsmöglichkeiten durchzutesten. Im Extremfall werden während der Kurzarbeit arbeitsorganisatorische und technologische Umstellungen vorbereitet bzw. vorgenommen, die dann in der nächsten Phase direkt zu Entlassungen und zu Arbeitsintensivierung für die Restbelegschaft führen (8). In solchen Fällen handelt es sich um die Finanzierung arbeitssparender Rationalisierungen durch die Belegschaften (8a).

Die Durchführung von Kurzarbeit geschieht entweder auf der Grundlage einer tarifvertraglichen Kurzarbeitsklausel (größtenteils in Manteltarifverträgen), einer Betriebsvereinbarung über Kurzarbeit oder unter Einbeziehung des Mitbestimmungsrechts des BR.

Als Kompensation zur Genehmigung der Kurzarbeit wurde häufig gefordert:

- vorherige Einsicht in die Wirtschaftssituation des Unternehmens,

- Lohnausgleich (tarifvertraglich abgesichert in der chemischen und mineralölverarbeitenden Industrie, teilweise durch Betriebsvereinbarungen, teilweise in Form betrieblich ausgehandelter Geldbeträge),

- Überstunden- und Einstellungsstopp (9).

Der BR wird seine Stellungnahme zur Kurzarbeit von der Einschätzung der wirtschaftlichen Entwicklung in der nächsten Phase abhängig machen. Einerseits will er vermeiden, daß Entlassungen durchgeführt werden, andererseits will er zu große Einkommenseinbußen vermeiden. Die durchschnittliche Einkommensverminderung beträgt bei $25 \%$ Arbeitszeitausfall $5-10 \%$ des Tariflohnes (unterhalb der Bemessungsgrundlage von $2.800 \mathrm{DM} / \mathrm{Monat}$ ). Hinzu kommen die Einkommensverringerungen aufgrund entgangener Überstunden/Sonderschichten, die nochmals $5.10 \%$ betragen, insgesamt also ca. $15 \%$.

Wichtig für die betrieblichen Auswirkungen der Kurzarbeit ist auch, daß zwar die spaltenden und individualisierenden Mechanismen nicht in dem Außmaß wie bei Entlassungen auftreten, der Druck auf die Belegschaft aber doch in die gleiche Richtung wirkt. „Die Unternehmer haben in vielen Betrieben die Belegschafgen gespalten. Anfänglich haben sie fast ausschließlich die Produktion kurzarbeiten lassen, fast immer wurden die Angestellten von der Kurzarbeit ausgenommen ... Die Arbeiter haben meistens dagegen protestiert, daß die Angestellten weiter arbeiten konnten, sie haben von den Betriebsräten verlangt, dafür zu sorgen, daßß auch die Angestellten in die Kurzarbeit einbezogen werden. Diese Praktiken haben oft die Angestellten gegen den Betriebsrat aufgebracht. ${ }^{\text {" }}(10)$

8 Vgl. am Beispiel OSRAM-Westberlin - Betriebspolitik zwischen Prosperität und Krise, in: PROKLA 23/1976, S. 101 ff., hier S. 112, 114 und 120.

8 a Eine andere Spielart des Mißbrauchs versuchte kürzlich die Klöckner-Hütte Bremen zu praktizieren. Sie stell te einen Antrag auf Kurzarbeit, als einer der Winderhitzer des Hochofens II explodierte und daher die Tagesleistung heruntergefahren werden mußte. Der $\mathrm{BR}$ forderte für seine Zustimmung vollen Lohnausgleich, die Einigungsstelle lehnte den Unternehmensantrag ab. Vgl. ausfuihrlich Arbeiterpolitik 6/76 v. 17.12.76, S. 19 f.

9 Vgl. z. B. Klaus Armann, Aus dem Wochenbuch eines Betriebsrates, in: Krise und Gegenwehr hg. von Rainer Duhm und Harald Wieser, Berlin 1975, S. 118

10 Arbeitsgruppe Ruhrgebiet, Unterdrückung im Betrieb, in: Links Nr. 73 v. Januar 76, S. 9 


\section{Personalabbau:}

Die Verringerung der Belegschaften in der Kfz-Industrie begann bereits 1971, verstärkte sich ab Herbst 1973 und fand erst Mitte 1975 ihr Ende. Bei der Mehrzahl der großen Automobilproduzenten wurden die Belegschaften um ca. $30 \%$ verringert (vgl. die Tabellen I - III).

\subsection{Einstellungsbeschränkungen:}

Der Erlaß von Einstellungssperren ist eine in allen Branchen verbreitete Maßnahme (von $37 \%$ aller befragten Betriebe angewandt). Sie wird spätestens gleichzeitig mit Kurzarbeit ergriffen. Allerdings handelte es sich in allen Fällen um sog. qualifizierte Einstellungsstopps, das heißt das Unterlassen des Fluktuationsersatzes nur bei bestimmten Beschäftigungsgruppen. Hier spielt das Argument vom Facharbeitermangel auch in der Krise eine große Rolle. Am stärksten betroffen waren die Arbeiter, insbesondere die un- und angelernten und unter ihnen die ausländischen Arbeiter.

Die Wirksamkeit dieser Maßnahme hängt weitgehend von der betrieblichen Fluktuationsrate ab. Sie betrug vor der Krise in einzelen Kfz-Unternehmen (einschl. der Kündigung durch das Unternehmen) bis zu $60 \%$, ging dann aber in der Krise generell zurück, wobei der Anteil der Unternehmerkündigungen rapide stieg. D. h., daß die sog. natürlichen Abgänge abnehmen und auch nicht den Selektionsvorstellungen der Unternehmen entsprechen (11). Daher haben diese in den letzten Jahren verstärkt auf neue Instrumente der Fluktuation hingearbeitet. Dazu gehören die vorzeitigen Pensionierungen und die Aufhebungsverträge (siehe dort) sowie die Zeitverträge insbesondere mit ausländischen Arbeitern. Das Problem der Notwendigkeit von innerbetrieblichen Umsetzungen bleibt aber bei diesen Instrumenten erhalten.

\subsection{Nichterneuerung von Zeitverträgen:}

Zeitverträge werden schon unter dem Gesichtspunkt abgeschlossen, bei Produktionseinschränkungen über ein konfliktlos zu handhabendes Abbaupotential zu verfügen. Betroffen davon waren maßgeblich ausländische Arbeiter aus Nicht-EG-Staaten.

Generell wird von Zeitverträgen häufiger in Klein- und Mittelbetrieben Gebrauch gemacht. In Großbetrieben werden sie normalerweise nur in Forschungs- und Entwicklungsabteilungen abgeschlossen. In anderen Bereichen sind sie auch gegen den Widerstand von Gewerkschaft und BR's kaum durchsetzbar.

In einem Fall wurden in der unsicheren Konjunktursituation Frühjahr 1975 alle Neueinstellungen nur mit Zeitverträgen vorgenommen, die dann insbesondere aufgrund der Aktivitäten des BR und der IGM verlängert und entfristet wurden (OpelRüsselsheim) (12).

11 ,Es zeigt sich nämlich, daß in Zeiten des konjunkturellen Abschwungs die sonst hohe Fluktuationsquote sinkt. Gerade leistungsschwache Mitarbeiter pochen dann auf vermeintlich wohlerworbene Rechte. Umgekehrt zeigt sich, daß gerade tüchtige Mitarbeiter trotz einer allgemein unsicher gewordenen Beschäftigungslage das Unternehmen verlassen, am auf einen Arbeitsplatz zu wechseln, der ihnen krisenfester erscheint. "Walter Schmidt, Personalanpassung in der Abschwungphase, in: Personal, Heft 6/74, S. 264 ff., hier S. 265. 


\subsection{Vorzeitige Pensionierung:}

Bei mindestens 59jährigen Frauen und 62jährigen Männern ist. Frïhpensionierung möglich. Von einigen Unternehmen wurden schon für 59 jährige Männer Aufhebungsverträge mit Überbrückungsgeld angeboten.

Entscheidend für das Unternehmen ist, daß über diese Maßnahme verbrauchte Arbeitskräfte abgeschoben werden können. Dadurch wird es nicht nötig, diese innerhalb des Unternehmens umzusetzen (auf sog. Schonarbeitsplätze), die damit verbundenen Konflikte auszutragen und zudem kann ein Teil der Kosten auf die BA abgeschoben werden. Die älteren Arbeitskräfte können dabei kaum mehr erreichen als finanzielle Ausgleichsleistungen, die sie nicht schlechter als im Fall einer „normalen“6 Pensionierung stellen.

Obwohl die Vorzeitpensionierung eine Abwälzung von Kosten auf die Rentenversicherung beinhal tet, bleibt die Kostenbelastung der Unternehmen i. V. zu anderen Abbauformen relativ hoch: „Die Zusatzbelastung bei einer um $5 \mathrm{~J}$ ahre vorgezogenen Rentenzahlung liegt damit grob gerechnet bei über 50000 .- DM." (13) Von Unternehmensseite wird argumentiert, daß man sich den ,heimlichen Übergang zur Altersgrenze 60 " kaum leisten könne und dieses Instrument ,möglichst wieder in der Versenkung versch winden lassen "solle (14).

Die Verrentung ist von staatlicher Seite abgesichert:

- ab 60 bzw. 65 Jahren bei 15 jähriger Versicherungszeit

- ab 60 Jahre, wer zusätzlich in den letzten eineinhalb Jahren mindestens 52 Wochen arbeitslos war (vorgezogenes Altersruhegeld wegen Arbeitslosigkeit);

- ab 62 Jahre für Schwerbehinderte, Berufsunfähigkeits- und Erwerbsunfähigkeitsrentner bei einer Wartezeit von 15 Jahren und 35 anrechnungsfähigen Jahren;

- ab 63 Jahre unter den gleichen Bedingungen (flexible Altersrente).

\subsection{Entlassungen:}

$48 \%$ der befragten Unternehmen der Stahl-, Maschinen- und Fahrzeugbauindustrie haben Entlassungen durchgeführt, dieses Instrument ist also neben dem Abbau von Überstunden das wichtigste, zeitlich diesem aber meist nachgelagert.

$\mathrm{Zu}$ Massenentlassungen ( $\$ 17 \mathrm{KSchG}$ ) ist es allerdings in keinem Fall gekommen. Die Unternehmen haben sich stets an der Untergrenze (49 Entlassungen pro Monat, sukzessive Entlassungen) gehalten. Daß ihnen dies möglich wurde, liegt weitgehend an der breiten Anwendung des Instruments Aufhebungsverträge mit Abfindungen (s. u.).

Für die entlassenden Unternehmen schließt die Entlassung einige Risiken in der Handhabung ein: die rechtlichen Schranken aufgrund von KSchG und BetrVG, der besondere Kündigungsschutz für bestimmte Beschäftigungsgruppen (ältere Arbeiter, Behinderte, werdende Mütter und Wöchnerinnen, Betriebsräte und Jugendvertreter), die zeitlichen Verzögerungen aufgrund der Kündigungsfristen, der mögliche Verlust von Ausbildungsinvestitionen und schließlich die Verschlechtenung des Unternehmens-

13 Heinz Struiber, Problematik der Personalanpassung, in: Personal, Heft 1/75, S. 2 ff., hier S. 3.

14 Ebenda 
Images auf dem Arbeitsmarkt.

Die Entlassungen wurden in den Unternehmen maßgeblich nach zwei Kriterien durchgeführt (15):

a. dem Senioritätsprinzip, d. h. der Differenzierung der Belegschaft nach der Dauer der Betriebszugehörigkeit. Die zuletzt eingestellten Belegschaftsmitglieder, d.h. Arbeitskräfte, die in der Hochkonjunkturphase zur Bewältigung vonProduktionsspitzen eingestellt wurden, werden bei Belegschaftsabbau zuerst entlassen.

b. dem Leistungsprinzip, d. h. der "Säuberung" der Belegschaft von leistungsschwachen Belegschaftsmitgliedern (unterdurchschnittliche Arbeitsleistung, hohe Fehlzeiten, oppositionelles Verhalten gegenüber dem Unternehmen etc.). So führte eines der befragten Unternehmen mit Billigung des BR eine Aktion ,müde Traber" durch.

Die Anwendung dieser Kriterien bedeutete gleichzeitig in den meisten Fällen eine zeitliche Reihenfolge, die gewährleistete, daß die Vorbehalte der Belegschaft gegenüber bestimmten Belegschaftsgruppen ausgenützt wurden und gleichzeitig ein Disziplinierungs- und Intensivierungsdruck auf die noch Beschäftigten ausgeübt wurde. So wurden zuerst Arbeitsverträge von Ausländern nicht verlängert, dann vereinzelte sauberungskündigungen vorgenommen (hohe Fehlzeiten, ,ewige Meckerer", „Säufer" etc.) und auch einzelne unbequeme Vertrauensleute und Gewerkschafter gekündigt. Dabei handelte es sich insbesondere um Kollegen, die in ihren Abteilungen den Widerstand gegen Personalabbau und Intensivierung zu organisieren suchten, auf Betriebsversammlungen auftraten und eine mit der Unternehmenspolitik konforme BR-Arbeit kritisierten. Mit solchen Entlassungen, die fast ausnahmslos die Zustimmung der BR fanden, war dann bei umfangreicheren Abbaumaßnahmen eine grundsätzlich ablehnende Haltung gegen Entlassungen nicht mehr möglich, die Belegschaft wurde verunsichert und inaktiv.

Die Entlassungen wurden häufig dadurch unauffällig durchgeführt, daß die Kündigungen am Wochenende übergeben und die betroffenen Kollegen für die 14 Tage Kündigungsfrist ab sofort beurlaubt wurden, sodąß sie von einem auf den anderen Tag aus dem Betrieb verschwanden (16)!

Die Haltung der BR schwankte zwischen der grundsätzlichen Ablehnung aller Entlassungen (die ja spätestens nach der Zustimmung der Einigungsstelle doch durchgefuihrt werden konnten), die maßgeblich von BR in Kleinbetrieben mit einem geringen gewerkschaftlichen Organisationsgrad eingenommen wurde. In Mittelund Großbetrieben wurde eher versucht, im Rahmen der arbeitsrechtlichen Möglich-

15 Diese Kriterien sind bereits das Ergebnis der betrieblichen Auseinandersetzung um die Selektion der zu Entlassenden. Insbesondere das Senioritätsprinzip dürfte für das Unternehmen nur unter bestimmten Gesichtspunkten wie Erhaltung betriebsspezifischer Qualifikation und Verstärkung der Betriebsbindung opportun sein; vgl. unten.

Nach einer Befragung von Industrieunternehmen in Baden-Württemberg im Sommer 1973 wurden folgende Kriterien als maßgebend bei Entlassungen bei einem Nachfragerückgang genannt: Leistung ( $57 \%$ aller Nennungen), Stillegung des entsprechenden Arbeitsplatzes ( $20 \%$ ), Ausbildungsstand der Arbeitskräfte $(16 \%)$, Nationalität $(0.8 \%)$. Auf die Bedeutung der Nationalität wird spärer noch eingegangen. Vgl. Siegfried Bullinger/ Peter Huber, Ausländerbeschäftigung aus Unternehmersicht, Tübingen 1974, S. 26 ff. Vgl z.B. Am Beispiel OSRAM-Westberlin, a.a.O., S. 112 
keiten Entlassungen zu verhindern, Kompensationen auszuhandeln (Sozialpläne, Abfindungen), den Kollegen Rechtsauskünfte zu geben und sie bei Arbeitsgerichtsprozessen zu unterstützen.

Dem Zwang zu Beteiligung an der Selektion können sich die BR - obwohl sie immer wieder den Schutz der betrieblich schwachen Belegschaftsgruppen betonen - weder bei der Einzelkündigung noch bei Mehrfachentlassungen entziehen. Häufig beantragten die Unternehmen übertriebene Entlassungszahlen, die die BR dann auf eine vom Unternehmen eingeplante Zahl reduzierten und dabei über die Sozialauswahl die Selektion vornahmen (das geschieht in bisher nur wenigen Betrieben aufgrund einer Betriebsvereinbarung entsprechend § 95 BetrVG - Auswahlrichtlinien). Seine Selektionskriterien stimmen dabei weitgehend mit denen der Unternehmen überein (Seniorität, Geschlecht, Familiensituation, Nationalität, Arbeitsleistung) (17) (18). Der entscheidende Unterschied liegt in der Betonung und weitgehenden Durchsetzung des Kriteriums Lebensalter durch die BR und damit der Berücksichtigung des Verschleißes der Arbeitskraft, deren Folgekosten die Unternehmen gerne abwälzen würden: durch Frühpensionierung, durch Entlassung kurz vor Erreichen der für verstärkten Schutz festgelegten Altersgrenze.

$17 \quad$ Zur Handhabung der Sozialauswahl nach KSchG liegt auch eine Grundsatzentscheidung des LAG Hamm vom 9.7.76 (3 Sa 141/76) vor. „Es sei allgemein anerkannt, daß die Frage, welche Arbeitnehmer eine Entlassung härter treffe, nur aufgrund einer gesamten Abwägung aller maßgebenden sozialen Umstände beantwortet werden könne. Dabei seien vor allem das Lebensalter, die Dauer der Betriebszugehörigkeit, der Familienstand, die wirtschaftliche Lage des Arbeitnehmers, insbesondere die Zahl der Unterhaltsberechtigten, aber auch der mitverdienenden Familienangehörigen und ähnliche Umstände zu berïcksichtigen." Zitiert nach Handelsblatt vom 3.11.76. „Gründe wie häufige Krankheit und eine schwächere Leistungsfähigkeit rechtfertigten eine Kündigung des Arbeitsverhältnisses nur dann, wenn diese bei verständiger Würdigung . . . angemessen sei. “In dieser konkreten Entscheidung wurde die Dauer der Betriebszugehörigkeit stärker gewertet.

18 Es liegt dann auch in der Konsequenz der innerbetrieblichen Selektion, wenn die Abfindungen nach den gleichen Kriterien abgestuft werden und z.B. auch der Unterstützungsverein der chemischen Industrie seine Leistungen von einer langen Betriebszugehörigkeit abhängig macht. Dieser Fond wurde im März 1975 von der IG Chemie als Tarifvertrag mit den Chemieverbänden mit dem Ziel abgeschlossen, die finanziellen Auswirkungen von Arbeitslosigkeit für Chemiearbeiter abzuschwächen. Voraussetzungen für die Zahlung eines Zuschusses von $15 \%$ des Arbeitslosengeldes zum Arbeitslosengeld sind:

- Entlassung aus betriebsbedingten oder unverschuldeten, personenbedingten Gründen;

- achtjährige, ununterbrochene Beschäftigung in der chemischen Industrie;

- ununterbrochene vierjährige Betriebszugehörigkeit;

- Arbeitslosigkeit von mehr als einem Monat,

- Bezug von Arbeitslosengeld,

- bezahlte Abfindungssummen werden abgerechnet.

(Vgl. ausführlich: Der Unterstützungsverein der chemischen Industrie, Erläuterungen und prakt ische Hinweise, Wiesbaden 1975, § 4).

Es liegt nicht an der unterdurchschnittlichen Entlassungsquote in der Chemieindustrie, sondern an den dermaßen eng gefaßten Bedingungen, wenn die gezahlten Beihilfen (bis 31.12.75 insgesamt 75 000 DM) niedriger als die Zinsen aus dem Fond-Vermögen sind (vgl Göttinger Betriebs Express vom 7.10.76). 
Die wesentlichen rechtlichen Eingriffsmöglichkeiten bestehen in den Bestimmungen des Kündigungsschutzgesetzes und des BetrVG. Generell ist der BR vor jeder Kündigung zu hören und über die Gründe zu informieren. Für die weitergehenden Rechte sind zwei Komplexe zu unterscheiden:

a. bei personen- und verhaltensbedingten Kündigungen kann er nicht widersprechen (außer bei einer Vereinbarung über ein generelles Widerspruchsrecht nach $\S 102$, Abs. 6 BetrVG), dem Betroffenen steht nur der Weg zum Arbeitsgericht offen.

Bei der betriebsbedingten Kündigung ist Widerspruch möglich, wenn sich der BR auf einen der Fälle des $\S 102$ Abs. 2 Nr. 1 - 5 BetrVG stützt:

- unzureichende Berücksichtigung sozialer Gesichtspunkte,

- Verstoß gegen eine Auswahlrichtlinie nach § 95 BetrVG,

- Möglichkeit der Weiterbeschäftigung des Betroffenen in einem anderen Betriebsoder Unternehmensteil,

- Weiterbeschäftigung nach Umschulung bzw. Fortbildung,

- Möglichkeit der Weiterbeschäftigung nach einverständlicher Vertragsänderung (Änderungskündigung § $2 \mathrm{KSchG}$ ).

b. bei der betriebsbedingten Kündigung (19) wird auf die Verhältnisse des Betriebes abgestellt. Sie muß notwendig und in dem Sinne dringend sein, als für die Weiterbeschäftigung „,betriebswirtschaftlich kein Bedarf ist und andere Gestaltungsmöglichkeiten organisatorischer Art . . . dem Unternehmen nicht zumutbar sind." Ursachen für betriebsbedingte Kündigungen können sowohl überbetriebliche (Rohstoffmangel, Absatzschwierigkeiten) wie betriebliche sein (Rationalisienung, Veränderung der Produktion, Einschränkung oder Aufgabe des Betriebs bzw. von Betriebsteilen). „Die Verbesserung der Ertragslage sowie die Kostensenkung im Betrieb sind grundsätzlich sachlich gerechtfertigte betriebliche Erfordernisse, die eine betriebsbedingte Kündigung zulassen." (ArbG Bremen vom 30.3.1967).

Bei Widerspruch des BR und einem folgenden Verfahren vor der Einigungsstelle ( $\$ 102$ Abs. 6 BetrVG) und Kündigungsschutzprozeß liegen weitgehend negative Erfahrungen vor. Abgesehen davon, daß der Gekündigte den langen Zeitraum und die Kosten des Verfahrens kaum überbrücken kann, ist dies im grundlegenden Standpunkt der Arbeitsgerichtsbarkeit begründet: „Die Handhabung dieses Kündigungsgrundes (aus dringenden betrieblichen Gründen, d. V.) durch die Arbeitsgerichte ist von dem Bestreben geprägt, die Dispositionen des Unternehmers nicht zu behindern und lediglich einzelne extreme unsoziale Konsequenzen zu korrigieren. Wichtigster, wenn auch nicht übermäßig häufig ausgesprochener Grundsatz ist die Ablehnung jeder sachlichen Überprüfung der zugrundeliegenden Unternehmerentscheidgung durch das Arbeitsgericht." (20) Ein Beispiel: „In einem weiteren uns bekannten Unternehmen sind in einem Zeitraum von 2 Monaten 32 Arbeiter und Angestellte gekündigt worden. 25 der Betroffenen haben über die zuständige Ortsverwaltung der IGM Kündigungsschutzklagen angestrengt. In zeitraubenden Arbeitsgerichtsprozessen konnte in keinem Fall eine Wiedereinstellung erzwungen werden, weil die Arbeitsgerichte

19 Vgl. ausführlich: Dirk Buchholtz, Betriebsbedingte Kündigung, Bergisch-Gladbach 1976, S. $33 \mathrm{ff}$.

Wolfgang Däubler, Das soziale Ideal des Bundesarbeitsgerichts, Frankfurt 1975, S. 104 
sich auf den Standpunkt stellten, daß bei Produktionseinschränkungen Entlassungen vorgenommen werden können. In diesen 25 Fällen hat das Arbeitsgericht Abfindungssummen von DM 800.-- bis DM 6 000.-- verfügt. Den Betrag von DM 800.-- hat ein türkischer Arbeiter bekommen, der zwei Jahre in dem Unternehmen tätig war." (21) An diesem Beispiel wird schon deutlich, weshalb Aufhebungsverträge für von Kündigung bedrohte Arbeiter attraktiver waren (höhere Abfindungssumme, kein Risiko des Entscheids, keine zeitliche Verzögerung).

Bei Widerspruch des BR aufgrund mangelnder Berücksichtigung der Sozialauswahl gerät er in die Situation, betriebliche Gruppen prinzipiell benachteiligen zu müssen und zwischen einzelnen Beschäftigten abzuwägen. Es ist deshalb nicht verwunderlich, daß die BR personenbedingte Kündigungen vorzogen, bei denen sie ihre Zustimmung durch "Verschulden" des Betroffenen legitimieren konnten und gleichzeitig die betrieblichen Auseinandersetzungen über Begründung und Außmaß ihres Widerstandes gegen Entlassungen verringern bzw. hinausschieben konnten.

Ein extremes Beipiel dafür ist die Stellungnahme des Opel-BR, ,daß gezielt in derÖffentlichkeit verbreitete Gerüch`e über Massenentlassungen bei Opel jeder Grundlage entbehren. Zur Zeit ist zwar die Entlassung von 49 Mitarbeitern geplant (die Zahl liegt unter der Meldepflicht für Massenentlassungen), doch sind die Gründe nicht in der wirtschalichen Lage, sondern in den Personen zu suchen." Eine höhere Arbeitsbelastung für die Restbelegschaft konnte der Opel-BR nicht sehen, da der Krankenstand um $5 \%$ gesunken sei - es war maßgeblich wegen hoher Krankenzeiten gekündigt worden (22).

Die betrieblichen Lernprozesse der BR und der Gewerkschaften drücken sich in Initiativen zur Verbesserung des Kündigungsschutzes aus. Die wesentlichenPunkte sind im Antrag 153 zum 10. DGB-Kongreß aufgenommen: „... Bei der Ausgestaltung des künftigen Arbeitsverhältnisrechts ist unter anderen folgendes zu beachten:

1. Die rechtliche Unterscheidung zwischen Arbeitern und Angestellten muß aufgehoben werden ...

2. Unter Beachtung der Bedeutung des Arbeisplatzes für alle Arbeitnehmer muß das Kündigungšrecht grundsätzlich umgestaltet werden. im einzelnen ist

a. eine Kündigung des Arbeitgebers grundsätzlich dann auszuschließen, wenn die demokratisch gewählte Interessenvertretung der Arbeitnehmer einer Kündigung widerspricht. In einem solchen Fall darf das Arbeitsverhältnis nur durch ein gestaltendes Gerichtsurteil aufgelöst werden.

b. Die Geltung des Kündigungsschutzes ist unabhängig vom Alter für alle Arbeitnehmer herzustellen. Für ältere Arbeitnehmer ist grundsätzlich die Unkündbarkeit vorzusehen

c. Dem Arbeitgeber ist die Beweislast dafür aufzuerlegen, daß er die richtige sozials Auswahl bei einer Kündigung getroffen hat ..."(23)

$21 \quad$ Arbeitsgruppe Ruhrgebiet, Unterdrïckung im Betrieb, a.a.O., S.10

22 Dokumentiert in: Opel-Bochum 1972-1975 - eine Belegschaft sammelt Erfahrungen, Bochum 1975, S. 70 ff.

23 DGB, Angenommene Anträge und Entschließungen des 10. ordentlichen Bundeskongresses vom 25. - 30. Mai 1975 in Hamburg, Düsseldorf 1975, S. 117 


\subsection{Aufhebungsverträge mit Abfindung (24):}

$\mathrm{Da} B$ es in der Automobilindustrie trotz der umfangreichen Belegschaftsverringerungen zu keinen Massenentlassungen kam, liegt am breiten Einsatz des Instruments der Aufhebungsverträge zumindest in den Großbetrieben. Welche Gründe die überragende Bedeutung gerade in dieser Branche hat, bleibt zu klären.

Neben dem Instrument der Kurzarbeit kam den Aufhebungsverträgen der gröBte Anpassungseffekt zu. Ca. $50 \%$ des Abbaus wurden hierüber getätigt (in einem Unternehmen über $72 \%$ ).

Aufhebungsverträge mit Abfindung sind befristete Angebote der Unternehmen an einen mehr oder weniger eng gefaßten Belegschaftsteil, auf ihre Rechte aus KSchG und BetrVG freiwillig zu verzichten und das durch eine finanzielle Abfindung kompensieren zu lassen. Sie beruhen auf den Prinzipien der Einvernehmlichkeit (zwischen Unternehmen und Einzelarbeiter) und der formellen Freiwilligkeit (der Annahme durch den Arbeiter/Angestellten). Ihrem Inhalt nach stehen sie zwischen Einzelkündigung und Interessenausgleich/Sozialplan. In Großbetrieben sind durchschnittlich folgende Bestimmungen in den Aufhebungsanträgen enthalten (25) (26):

Michael Kittner, IGM, erweitert diese Forderungen: ,Zu einer Erweiterung von Mitbestimmungsrechten (des BR) müßte wahrscheinlich eine Kombination von Einstellungsgeboten plus Abgabenpflicht bei deren Nichterfüllung auf der einen und Subventionierung aus den so angesammelten Geldern auf der anderen Seite treten." (FR vom 22.12. 76, S. 13)

24 Vgl auch Hans Gerhard Mendius/Rainer Schultz-Wild, Personalabbau und Interessenvertretung durch den Betriebsrat, Manuskript für Leviathan 4/76

Zur formalen Durchführung durch das Unternehmen vgl. Mart in Posth/Rainer Haßheider, Personalabbau - Abschied mit Abfindungen, in: Personal, Heft 8/74, S. 346 ff.

25 Die erste Aufhebungsaktion in der VW-AG zum 1.1.74 betraf ca. 3500 Arbeiter, denen Abfindungen $\mathrm{zw}$ ischen 5000 .- und 9000.- DM gezahlt wurden, die sich zusammensetten aus:

- Erfolgsprämie für 1973 (7\% des Jahresbruttoverdienstes)

- tarifliches Urlaubsgeld

- Jahresurlaubsanspruch und

- ein zusätzlicher Monats!ohn.

Bei der Bewertung der Abfindungen ist zu berücksichtigen, daß nur der zusätzliche Monatslohn eine Abfindung darstellte, die zudem bei anschließender Arbeitslosigkeit durch eine Sperrfrist beim Bezug von Arbeitslosengeld aufgebraucht wurde. Abgefundene Arbeiter, die länger als 14 Jahre bei $\mathrm{VW}$ beschäftigt waren, verloren zudem ihre Ansprüche auf die Werksrente. Vgl. ausführlich: Klaus Gülden/ Horst Peter, VW: Krisenlösung durch Entlassungen, in: Gewerkschaft und Klassenkampf, Kritisches Jahrbuch '75, S. $41 \mathrm{f}$.

26 Der Anspruch auf Arbeitslosengel $;$ ruht nach $\S 117$, Abs. 2 des AFG, wenn der Arbeitslose wegen Beendigung des Arbeitsverhältnisses eine Abfindung, Entschädigung oder ähnliche Leistungen erhalten oder $z u$ beanspruchen hat und das Arbeitsverhältnis durch Aufhebungsvertrag oder Vergleich beendet wurde. Der Anspruch ruht nur dann nicht, wenn der Betroffene diese Leistungen auch bei ordentlicher Kündigung hätte beanspruchen können. Andernfalls ruht der Anspruch für die Dauer der Kündigungsfrist. Vgl. Handelsblatt vom 25.11.75.

Nach einem Grundsatzbeschluß des Bundesverfassungsgerichts vom Juli 76 darf diese Bestimmung aber nicht dazu führen, daß der Betroffene seine gesamte Abfindung einschließlich ihres sozialen Bestandteils, der als Ausgleich für die Einbuße an sozialem Besitzstand durch Arbeitsplatzverlust steht, durch das Ruhen des Arbeitslosengeldes aufbrauchen muß. Vgl. Handelsblatt vom 2.8.76. 
a. eine Barabfindung (oder ein Zuschuß zur Altersversicherung bei Vorzeitpensionierung), deren Höhe als unterschiedliche Zahl von Monatsentgelten vereinbart wurde (die Untergrenze konnte für Nicht-EG-Ausländer aber auch unter einem Monatsgehalt liegen). Die Höhe der Abfindung ist in der Regel gestaffelt nach der Dauer der Betriebszugehörigkeit, Einkommenshöhe und manchmal dem Alter.

Eine solche Differenzierung benachteiligte die jüngeren und einkommensschwachen Belegschaftsgruppen, stand somit in umgekehrten Verhältnis zum Arbeitsplatzrisiko.

b. Die Sicherung von Sonderleistungen des Unternehmens über das Ausscheiden hinaus (Werksrente, Werkswohnung, verbilligter Bezug von Unternehmensprodukten etc.)

c. Eingeschränkte Zusagen der Wiederbeschäftigung der Abgefundenen bei Neueinstellungen.

Abfindungsaktionen wurden als Betriebsvereinbarung ( $§ 77$ BetrVG) zwischen Unternehmen und BR ausgehandelt. Die Initiative ging in allen Fällen vom Unternehmen aus.

Wie schon festgestellt, wird dieses Instrument von den Unternehmen vor dem Hintergrund der Alternative von Massenentlassungen beurteilt. Deren Kosten sind

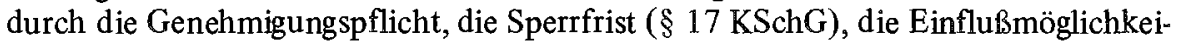
ten des BR und der Arbeitsgerichte, mögliche Entschädigungsleistungen, Sozialpläne etc. sehr hoch. Demgegenüber hat das Instrument der Aufhebungsverträge einen kürzeren Realisierungszeitraum, eine bessere Planbarkeit, ein geringeres soziales und politisches Konfliktpotential im Betrieb und gegenüber der Öffentlichkeit. Nachteilig wird die Verletzung des Prinzips vermerkt, ,,wonach man gute Mitarbeiter durch Zusatzleistungen an den Betrieb binden, nicht aber den Abgängern noch etwas hinterherwerfen soll." (27) Zudem wird eine präjudizierende Wirkung dahingehend gesehen, daß in Zukunft betrieblich bedingte Kündigungen kaum noch ohne Abfindungen durchgeführt werden können (28).

Die Steuerbarkeit ist gegeben erstens durch die Möglichkeit, mehrere Aufhebungsaktionen jeweils nach ihrem Erfolg zu staffeln. Zweitens durch die zeitliche Befristung des Angebots, drittens durch die Festlegung der Zahl der angebotenen Aufhebungsverträge, viertens durch die Festlegung von Quoten für Arbeiter und Angestellte und für die einzelnen Lohngruppen. Trotz dieser Einschränkungen kann noch in größerem Maß eintreten, daß die formell gewährte Freiwilligkeit der Annahme die Selektionsvorstellungen des Unternehmens sprengt, d. h., daß Arbeitskräfte kündigen, die gehalten werden sollen (insbesondere Facharbeiterhortung) und umgekehrt viele Arbeitskräfte die Verträge nicht annehmen auf die die Aktion gezielt war. Um diesen Spielraum einzuschränken, besteht erstens die Möglichkeit, mit dem BR eine Einvernehmlichkeitsklausel zu vereinbaren, nach der dem Unternehmen fallweise ein Rückzugsrecht des Angebots zusteht. Zweitens kann es auf individuelle Aufhebungsverträge umstellen, wie das der Ausschuß ,Probleme bei Personalein-

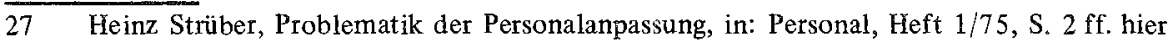
S. 3

28 Ebenda, S. 4 
schränkungen" der Deutschen Gesellschaft für Personalführung empfiehlt (29) und wie es anscheinend in vielen Branchen massenhaft gehandhabt wurde, ohne daß relativ spektakuläre Aufhebungsaktionen durchgeführt wurden (30). In beiden Fällen wird eine Steuerung auch indirekt dadurch bewirkt, indem bestimmten Beschäftigtengruppen vordringlich Aufhebungsverträge angeboten bzw. dies bei bestimmten Gruppen abgelehnt werden. Außerdem werden die angezielten Arbeitskräfte dadurch „motiviert", daß sie bei fehlender Freiwilligkeit anschließend normal entlassen würden mit geringeren Gratifikationen.

Genau an diesem Punkt setzt das Interesse des BR ein: er wird versuchen, das Unternehmensangebot über die Kompensationen bei normalen Kündigungen hinauszudrücken. Dabei steht ihm kein formales Recht zu, sondern sein Einfluß gründet sich auf den Einfluß bei andernfalls notwendigen Entlassungsformen, den das Unternehmen gern vermindern würde. Insofern liegt es sehr im Unternehmerinteresse, sich mit dem BR auf ein mittleres Abfindungsvolumen zu einigen (31).

Die BR hatten generell Schwierigkeiten, da das Instrument für sie neu war und die Folgewirkungen kaum zu übersehen waren; zudem standen sie unter Zeitdruck. Ihre Hauptlinie bestand darin, die Auswirkungen der ihnen notwendig erscheinenden Entlassungen zu mindern. Bei Vorzeitpensionierungen berieten die BR die Belegschaftsmitglieder meist in positivem Sinne, wobei sie sich im Klaren waren, daß sie damit das Unternehmen der Notwendigkeit enthoben, Arbeitsplätze für ältere Arbeitskräfte zu gestalten und generell die Arbeitsbelastung so zu dimensionieren, daß das Arbeitsvermögen nicht geschädigt wird; weiterhin, daß ein Teil der Folgekosten übermäßiger Arbeitsabpressung in die Rentenversicherung umverteilt wird.

Bei den anderen Aufhebungsverträgen war die Position der BR widersprüchlicher, sodaß die Entscheidung den größtenteils schlecht informierten Arbeitern individuell überlassen wurde. In der Frage, ob die Aufhebungsaktionen offen für alle Belegschaftsmitglieder gestaltet werden sollten, existierten im Extrem zwei Positionen. Eine plädierte für eine genaue Selektion, um durch das Ausscheiden genau der angezielten Belegschaftsteile die Betriebsrentabilität zu steigern und damit die verbliebenen Arbeitsplätze zu sichern. Die andere Position ging von einem Versagen der Unternehmenspolitik aus, das Entlassungen verursacheund daß dann wenigstens allen Belegschaftsmitgliedern die Entscheidung über ihr Verbleiben im Betrieb zugestanden werden müsse. Außerdem werde dadurch vermieden, daß ein gezielter Druck zur Kündigung auf bestimmte Belegschaftsteile ausgeübt werde.

29 Vgl. Helmut Glaubrecht, Probleme bei Personaleinschränkung, in: Personalprobleme im Konjunkturverlauf, Handstein Verlag Köln, 1976, S. 18

30. Bei dieser sogenannten Auflösung des Arbeitsverhältnisses im beiderseitigen Einverständnis stehen dem BR keine Rechte und direkten Einflußmöglichkeiten zu, es besteht allerdings die Tendenz, den BR stärker in die Verant wortung miteinzubeziehen.

31 Eine rechtlich noch nicht entschiedene Einflußmöglichkeit des BR bei Massenentlassungen wäre dann gegeben, wenn der Tatbestand einer „Betriebsänderung “ anerkannt wird, ,die wesentliche Nachteile für die Belegschaft oder erhebliche Teile der Belegschaft zur Folge haben" muß ( $\$ 111$ BetrVG). Aus ihr folgt die Verpflichtung zur Aushandlung eines Interessenausgleichs und eines Sozialplans ( $\$ 112$ BetrVG). 


\section{Exkurs: Die verdeckte Selektion bei Aufhebungsverträgen}

Die Aufhebungsaktionen in der Automobilindustrie haben gezeigt, daß die angezielten Belegschaftsgruppen - weitgehend un- und angelernte Arbeiter, insbesondere ausländische Arbeiter - diese Aufhebungsangebote auch freiwillig angenommen haben. Was als Interessenidentität von Arbeitern und Unternehmen erscheint, verbirgt die einer solchen Entscheidung vorgelagerten und sie einbettenden Selekt ionsmechanismen. In Wirklichkeit haben un- und angelernte Arbeiter ein überdurchschnittliches Interesse an einem sicheren Arbeitsplatz, da es ihnen nur bei längerer Betriebszugehörigkeit gelingen kann, ihre Qualifikation und ihr Einkommen zu erhöhen, in den Genuß der an die Dauer der Betriebszugehörigkeit gebundenen Rechte und Gratifikationen zu kommen, und da ihre Möglichkeiten, einen neuen Arbeitsplatz zu finden, überdurchschnittlich eingeschränkt sind, Von daher wäre es logisch, wenn gerade sie nicht auf solche Aufhebungsaktionen eingingen. Zur Erklärung ihres Verhaltens müssen mehrere Faktoren herangezogen werden. Ihr Verbleiben im Betrieb könnte nur gesichert werden, wenn seitens des Unternehmens ein explizites Interesse an ihrem Verbleiben bestünde, wenn ausgebaute Rechtsschranken gegen ihre Kündigung existierten und ihre Stellung in der betrieblich/gewerkschaftlichen Interessenvertretung stark wäre. Keiner dieser Faktoren ist gegeben. Dem steht nicht entgegen, daß formalrechtlich die arbeitsrechtliche Gleichstellung und seine Vertretung durch den BR abgesichert ist.

Das Unternehmen will Arbeitskräfte loswerden insbesondere im Produktionsbereich; Ungelernte und Angelernte sind leicht zu ersetzen (Druck der industriellen Reservearmee), sie verfügen nur über eine geringe allgemeine und betriebliche Qualifikation, die in einer Anlernphase vielen anderen Arbeitskräften auch zu vermitteln ist. Ähnliches gilt für die qualifizierteren älteren Arbeitskräfte, deren Spezialqualifikation das geschwundene Arbeitsvermögen nicht mehr kompensieren kann.

Die arbeitsrechtlichen Schutzbestimmungen sind für jüngere, gering qualifizierte Arbeitskräfte am geringsten ausgebaut, da ihr sozialer Besitzstand, der zu schützen wäre, vergleichsweise am geringsten ist (Kündigung, Umsetzung etc.). Am schlechtesten sind hier die Arbeitsimmigranten gestellt, am besten die älteren Arbeitskräfte.

Die betrieblich/gewerkschaftliche Interessenvertretung wie auch die staatliche Arbeitsmarktpolitik (32) orientiert sich traditionell am Facharbeiterbild. Bei der Sozialauswahl stehen Kriterien wie Seniorität, Alter, Arbeitsleistung (stark an Qualifikation geknüpft) Geschlecht, Familienstand und Nationalität im Vordergrund. Mit dem Hinweis auf ihre unverbrauchte Lernund Arbeitsfähigkeit, ihre Mobilität werden aus dem akzeptierten Selektionszwang heraus genau die Voraussetzungen zu Qualifikation und Einkommenssteigerung für Un- und Angelernte beseitigt (Arbeitsplatzsicherheit).

Aus diesen Faktoren heraus ergibt sich eine permanente Bedrohungskonstellation für Un- und Angelernte, der sie individuell nicht begegnen können. Im Fall des Belegschaftsabbaus können sie nur versuchen, individuell so viel wie möglich herauszuholen und genau diese Möglichkeit spiegelt die Aufhebungskündigung mit Abfindung vor. $\mathrm{Zu}$ der allgemeinen Konstellation kommen aber noch konkrete Bedrohungsmechanismen hinzu, die kurz am Beispiel des VW-Konzerns beschrieben werden sollen (33).

32 Vgl. Lutz/Sengenberger, Arbeitsmarktstrukturen und öffentliche Arbeitsmarktpolitik, Göttingen 1974, S. 102 ff.

33 Die erste Aufhebungsaktion bei VW fand schon 1972 statt, bei der Abfindungen von $3.500,-$ bis $6.000,-$ DM geboten wurden. 1973/74 findet dann die sogenannte LeidingAktion statt, die Einstellung von 13.000 Produktionsarbeitern. Im Juni 74 dann das erste Aufhebungsangebot in der Krise, das 3.481 Arbeiter annahmen; im Oktober 74 das zweite Aufhebungsangebot für 6.000 Arbeiter und Angestellte mit Prämien von $5.000,-$ bis 9.000,- DM. Am 15.4.75 dann der Sanierungsplan, der einen Personalabbau von über 15000 vorsah, davon 5000 über Aufhebungsverträge. Die Abfindungen wurden auf $9000 .-$ bis $14700 .-$ DM (bei über 10-jähriger Betriebszugehörigkeit) erhöht.

Die ersten Neueinstellungen erfolgten im Oktober 75 mit 450 Akkord-Arbeitern in den Werken Wolfsburg und Emden, die ersten Sonderschichten wurden im April 76 vereinbart (12 Sonderschichten bis zum März 77, die aber schon im November 76 voll ausgenutzt waren). 
Der VW-Konzern hat zwischen Anfang 1974 und Herbst 1975 seine Belegschaft in der BRD um ca. $32000=25.4 \%$ verringert; bis zum Jahresende 74 hatten davon über $70 \%$ Aufhebungsverträge angenommen. Die Aufhebungsaktionen waren durch verschiedene Maßnahmen vorbereitet (34):

1. Seit 1974 wurde mehr oder weniger offen von der Notwendigkeit von Massenentlassungen gesprochen In der Unternehmenszeitung hieß es: „Weitergehende Schritte sind dann unerläßlich, wenn Aufhebungsverträge und Fluktuationsabgang allein nicht ausreichen ... Für die Mitarbeiter, deren Aufgaben wegfallen, ist es sicherlich erwägenswert, freiwillig zu einem früheren Zeitpunkt mit einer klar übersehbaren Förderungssumme versehen das Unternehmen zu verlassen, als sich später in die Unsicherheit eines möglicherweise notwendig werdenden Kündigungsverfahrens hineinzubegeben."

2. Wesentlich deutlicher und selektiver waren die Androhungen individueller Entlassung, die ,Mitarbeitern' ${ }^{6}$ Z.B. im Gespräch mit dem Meister übermittelt wurden. Bei diesem Konkretionsgrad der Entlassungsdrohung und fehlender Widerstandsperspektive bedeutete das für viele den direkten Zwang zur Annahme des Aufhebungsangebots. So wurden allein im Werk Hannover im April 1975 nach Schätzungen ca. 300 Arbeiter vom Vorteil der Aufhebungsverträge überzeugt.

3. Die gesamte Entlassungsaktion bei VW war von zahlreichen inner-und zwischenbetrieblichen Umsetzungen begleitet. Abgesehen von Einkommens- und Qualifikationsverlust (s.u.) wird damit häufig die Entlassung vorbereitet. Der Umgesetzte wird aus dem vertrauten Kreis von Kollegen und Vertrauensleuten herausgerissen und damit aus dem Solidaritätszusammenhang im Entlassungsfall. Er ,verschwindet“ erst in einer anderen Abteilung, in der er diese Kontakte nicht hat; wenn er dann ganz aus dem Betrieb verschwindet, wird es kaum noch bemerkt (35). Zum anderen ist der Umgesetzte in der neuen Abteilung der Neue, der auch als Erster wieder abgeschoben werden kann, wenn die Abteilung verringert werden soll. Mehrmalige Umsetzungen können die Betroffenen also derart aus ihrem menschlichen und Arbeitszusammenhang reißen, daß ihnen der Aufhebungsvertrag noch als günstige Absprungmöglichkeit erscheint. Ein wechselnder Arbeitsplatz im Unternehmen ist häufig die Vorstufe zum Arbeitsplatzverlust.

Die gesellschaftliche Situation der Un- und Angelernten, ihre Position auf dem Arbeitsmarkt und dann die ,vorbereitenden" betrieblichen Maßnahmen erklären also sehr wohl, weshalb die gefährdetsten Arbeitergruppen ,freiwillig“ Authebungsverträge annehmen (36). Bei VW waren

34 Vgl dazu Rainer Dombois, Massenentlassungen bei VW:Individualisierung der Krise, Manuskript für Leviathan 4/76

35 ,Die spielen die Angst richtig aus ... Als wir im Rohbau z.B. aus Materialgründen mit dem Programm von 290 auf 200 zurückgegangen sind, da hat der Ingenieur einfach an 8 Arbeitsplätzen, ohne mit dem Betriebsrat das abzusprechen, Leute aus der Produktion rausgezogen. Die wurden mit einer anderen Aufgabe beschäftigt. Diese 8 Mann sind noch nicht weg, wenn sie heute 290 fahren, müssen die da wieder arbeiten. Solang machen die was anderes, die fegen oder sonst irgendwas; die kosten VW im Moment noch genausoviel Geld. Aber wenn jetzt der eine davon kündigt, dann wird er nicht mehr ersetzt, weil er sowieso nur noch so nebenher beschäftigt wurde. Die sind meist nicht ganz weg, in eine andere Halle, in eine andere Meisterschaft, die gerade angerufen hat: hab heute zu hohe Abweserheit, ich brauche Leute. Die gehen durch die ganze Halle und dann siehst du sie ein paar Tage nicht, und die anderen Kumpels gewöhnen sich schon langsam daran, daß sie weg sind " Zitiert nach Günter Wallraff, Volkswagen. Eine Konzernstrategie und ihre Folgen, in: Krise und Gegenwehr, S. $88 \mathrm{f}$.

36 Gülden/Peter bestätigen die beschriebenen Unternehmenstechniken zur Vorselektion: "Man nutzte das Bestreben von Meistern und Abteilungsleitern aus, in ihren Abteilungen möglichst viel einzusparen und deutete einigen Kollegen unverhohlen an, ein frei- 
das maßgeblich die ausländischen Arbeiter (im Werk Hannover reduzierte sich die Zahl der Ausländer um über $70 \%$ fast ausschließlich über Aufhebungsverträge), die 13000 mit der ,LeidingAktion" 1973/74 Neueingestellten und die Pendler.

Der hohe Anteil der Ausländer bei den Aufhebungsverträgen ist in ihrer noch zusätzlich eingeschränkten Rechtsposition, der Durchsetzung des Inländerprimats durch die Arbeitsämter und ihrer Randexistenz in Betrieb und Interessenvertretung begründet (s. u.). Im Werk Hannover nahmen zwischen Oktober 74 und Juli 75 ca. $61 \%$ der dort beschäftigten Ausländer Aufhebungsverträge an, gegenüber $17.1 \%$ der deutschen Beschäftigten (37).

\section{Innerbetriebliche Versetzungen, Umgnuppienungen und Änderungskündigun- gen}

Im Zusammenhang mit beschäftigungswirksamen Maßnahmen erfolgen innerbetriebliche Umsetzungen meist nach Aufhebungsverträgen, da diese Maßnahme noch am wenigsten vom Unternehmen zu steuern ist.Abgesehen von den Ausnahmen, in denen noch benötigte, qualifizierte Arbeitskräfte diese Verträge angenommen haben, sind im Durchschnitt soviel unqualifiziertere Arbeitskräfte abgegangen, daß bei der Aufrechterhaltung der Belegschaftsstruktur nach Tätigkeitsanforderungen etliche der höher Qualifizierten herabgestuft werden müssen. Umsetzung in der Krise bedeutet dementsprechend auch: Verlust der alten, qualifizierteren Tätigkeit, der gewohnten Arbeitsumgebung und der arbeitsplatzspezifischen Qualifikation, weiterhin aufgrund der Herabstufung eine Verringerung des Einkommens. Zielgruppe solcher Umsetzungen waren maßgeblich Facharbeiter und Angestellte, die in die Produktion versetzt wurden (38).

Der quantitative Umfang solcher Umsetzung war in allen Automobilbetrieben erheblich; in einem der befragten Unternehmen betraf sie 5000 , in einem anderen 1000 Beschäftigte.

Bei Umgruppierungen und Versetzungen steht dem BR ein Mitbestimmungsrecht nach $\S 99$ BetrVG in Verbindung mit $\S 95 \mathrm{Abs} .3 \mathrm{zu}$, eine Vermittlungsfunktion beim Einspruch gegen eine Änderungskündigung ( $\$ 2 \mathrm{KSchG}$ ) (39). In der Regel versuchten die BR's, einen möglichst lang befristeten Lohnausgleich auszuhandeln (z.B. bis zu 24 Monaten). Sie gingen dabei davon aus, daß die Herabstufungen vorübergehend seien und mit dem zu erwartenden Aufschwung rückgängig gemacht werden. Viele Unternehmen schrumpften aber langfristig und machten das auch

williges Ausscheiden mit einer Abfindung wäre für sie günstiger als eine mögliche Entlassung nach den Betriebsferien. Das ging sogar soweit, daß in Einzelfällen Schwerbeschädigte wegen eines ,Aufhebungsvertrages' von Vorgesetzten angesprochen wurden. Andererseits kam es aber auch vor, daß Vorgesetzte versuchten, besonders Facharbeiter und Spezialisten von einem ,Aufhebungsvertrag' abzuhalten." a.a.O., S. 42.

Ebenso Express 4/74, S. 5

37 Vgl. Dombois, a.a.O., S. 17

38 VgL z.B. Informationen der Plakat-Gruppe/Stuttgart v. 3.12.75

39 Wann eine Änderungskündigung vorliegt, bestimmt sich nach Tarifvertrag (z.B. Wechsel der Lohngruppe). Wichtig für die Eingriffsrechte des BR sind weiterhin Einzelarbeitsvertrag, Arbeitsordnung und eventuell bestehende Betriebsvereinbarungen. 
durch eine schnelle Angleichung der Löhne an die neue Tätigkeit, d.h. Einkommensminderung, deutlich.

In einigen Tarifbereichen bestehen Regelungen, die die Möglichkeiten der Änderungskündigung ab einem bestimmten Lebensalter (z.B. MTV Metallindustrie in Nürdwürttemberg/Nordbaden ab 53 Jahre) erschweren und die Verdiensteinbußen begrenzen.

Die Anpassung der Personalstruktur an ein nur verringertes Produktionsvolumen nach Entlassungen/Abfindungsaktionen ist nur in wenigen Fällen das alleinige Motiv von Versetzungen. In der Mehrzahl der Fälle dürften sie mit längerfristigen Umorganisationen der Produktion einhergehen, die die Umsetzung und Herabstufung festschreiben. Die bisherigen Formen von Rationalisierungsschutzverträgen greifen hier überhaupt nicht.

Darüberhinaus sind Fälle bekannt geworden, bei denen rechtlich oder personalpolitisch unmögliche Entlassungen über eine oder mehrere Versetzungen durchgesetzt wurden. Der Betroffene verliert seinen attraktiven Arbeitsplatz und sein Lohnniveau, die seine Gegenwehr gegen Entlassung begründet haben; er verliert darüberhinaus den in langer Betriebszugehörigkeit erworbenen Kontakt zu seinen Arbeitskollegen, wird dadurch in seinen Einflußmöglichkeiten beschnitten und so für die Eigenkündigung „weichgemacht“ (40). Viele Facharbeiter akzeptieren daher eher eine Lohnminderung am alten Arbeitsplatz und beugen sich nur Strafumsetzungen an Akkordarbeitsplätze.

Eine weitere Ursache von Umsetzungen ist die verringerte Leistungsfähigkeit aufgrund gesundheitlichen Verschleißes im Alter. Wie sich aus Gesundheitsberichten und Betriebsstatistiken entnehmen läßt, nimmt die Zahl der sogenannten Minderleister aufgrund langfristig überhöhter Arbeitsverausgabung ständig zu. Ihre Situation wird in der Krise aus zwei Gründen noch erschwert: die Auslese nach dem Leistungsprinzip wird noch verschärft und viele der unterdurchschnittlich produktiven sogenannten Schonarbeitsplätze (z.B. Warenlager) werden wegrationalisiert. Die Unternehmensstrategie des Fixkostenabbaus zielt insbesondere auf solche Beschäftigtengruppen. Die hohe Arbeitslosenquote bei Schwerbeschädigten - trotz besonderen Kündigungsschutzes und staatlicher Lohnsubvention - ist Ausdruck der rigiden Durchsetzung des unternehmerischen Rentabilitätsprinzips.

40 ". . . wird die Versetzung oder Umgruppierung dazu benutzt, den Widerstand des Betriebsrates . . . zu unterlaufen ... Ein Reparaturschlosser soll entlassen werden. Dem Betriebsrat wird ein Entlassungsantrag zugleitet mit der Begründung ,Arbeitsmangel". Der Betriebsrat verweigert seine Zustimmung. Darauf bekommt der Reparaturschlosser, der bisher 11.- DM verdient hat, eine Änderungskündigung, in der mitgeteilt wird, $\mathrm{daß}$ er nun als Stapelfahrer eingesetzt wird; der neue Lohn beträgt DM 8.20 ... Gegen dieses Vorgehen der Unternehmer hat der Arbeiter das Recht des Einspruchs; der Einspruch wird in der Regel abgelehnt. Die Erfahrungen lehren, daß in solchen Fällen Klagen beim Arbeitsgericht kaum Erfolg haben ... Da diese Änderungskündigungen erhebliche Lohnverluste bedeuten, sagen nicht wenige Kollegen, ich laß mich lieber rauswerfen, dann bekomme ich für meinen jetzigen Lohn wenigstens noch ein hohes Arbeitslosengeld." Arbeitsgruppe Ruhrgebiet, a.a.O., S. 11 


\section{Relative Bedeutung der einzelnen Maßnahmen:}

Aus der folgenden Tabelle IV geht hervor, wieviel der befragten Betriebe in welcher Branche zu den einzelnen Maßnahmen gegriffen haben. Darin ist aber ihre Bedeutung für die Veränderung des betrieblichen Arbeitspotentials nicht adäquat ausgedrückt.

Tabelle 4: Personalpolitische Maßnahmen in den Jahren 1974/75 (in \% aller befragten Betriebe)

Gesamt Energie Verarb. Bau- Handel Verkehr, Kredit Dienstl. Wasserv. Gewerbe gewerbe Nachricht. instit. freie Bergbau übermittlg. Versich. Berufe

Reduzierung von

Überstunden

Abbau v. Sonder-

schich ten

Kurzarbeit

Entlassungen

Aufhebungsverträge

Vorz tge. Pensiong.

Einstellungssperre

Sozialplan erstellt

$\begin{array}{rrrr}66 & 43 & 76 & 76 \\ 12 & 2 & 20 & 10 \\ 30 & 1 & 44 & 33 \\ 49 & 7 & 54 & 63 \\ 5 & 3 & 5 & 9 \\ 13 & 9 & 16 & 12 \\ 37 & 24 & 45 & 31 \\ 6 & 8 & 7 & 5\end{array}$

$\begin{array}{rr}51 & 53 \\ 6 & 9 \\ 8 & 4 \\ 44 & 16 \\ 3 & 2 \\ 11 & 10 \\ 29 & 23 \\ 3 & 5\end{array}$

$32 \quad 34$

Quelle: Befragung von Groß betrieben durch das IFS München, vgl. Arbeitsbericht IV, a.a.O., S. 24

In der zeitlichen Aufeinanderfolge der einzelnen Maßnahmen zeigt sich ein ziemlich einheitliches Grundmuster: ,In jedem Falle wurden Sonderschich ten zuerst eingestellt, es folgten Einstellungsbeschränkungen und Kurzarbeit; während mit Abfindungen und vorzeitigen Pensionierungen deutlich später begonnen wurde." (40 a) Umgekehrt wurde Kurzarbeit vor anderen Maßnahmen eingestelit, die Authebungsaktionen liefen teilweise sehr lange.

Der zeitlichen Reihenfolge kommt auch insofern Bedeutung zu, als sie stufenweise die Bedrohung der Belegschaft steigern und damit parallel die kollektivbetrieblichen Widerstandsformen schwächen. Die Akzeptierung einer Maßnahme beinhaltet fast schon die Hinnahme der nächsten, schärferen Maßnahme.

\section{Personelle Schwerpunkte der Entlassungen}

Wie aus Tabelle I abzulesen ist, wurde die Zahl der Beschäftigten im Straßenfahrzeugbau insgesamt um $11.7 \%$ zwischen Oktober 73 und Dezember 75 verringert. Als Selektionskriterien resultierten aus der Auseinandersetzung zwischen Unternehmensleitung und BR maßgeblich:

(1) Dauer der Betriebszugehörigkeit und Alter

(2) Gesundheitliche und altersbedingte Beeinträchtigungen des Leistungsvermögens

(3) Arbeitsplatzbezogene, gewerkschaftliche und politische Aktivitäten im Be-

40 a ISF München, Arbeitsbericht IX, a.a.O., S. 109 
trieb.

Als Ergebnis betrieblicher Entlassungspolitik zeigte sich, daß das flexibelste Potential der Betriebe in den ausländischen Arbeitern bestand.'Da aufgrund der Umstellung der Arbeitsstatistik keine Globalzahlen über die Verringerung der Ausländerbeschäftigung in den Branchen seit dem Höhepunkt der Ausländerbeschäftigung im Herbst 1973 existieren, kann der Umfang der Abwälzung der Entlassungen auf ausländische Arbeiter nur an (allerdings sehr prägnanten) Einzelbeispielen abgeschätzt werden $(40 \mathrm{~b})$. Die IFS-Untersuchung gibt für zwei große Unternehmen der Automobilindustrie folgende Verteilung an:

Tabelle 5: Abbau verschiedener Beschäftigtengruppen in zwei Unternehmen der Automobilindustrie in der Phase des hauptsächlichen Personalabbaus 1973/1975 (in \% des jeweiligen Belegschaftsanteils)

$\begin{array}{lcc} & \text { Unternehmen 1 } & \text { Unternehmen } 2 \\ \text { Belegschaft insgesamt } & -22,8 & -25,9 \\ \text { Gehaltsempfänger } & -6,7 & -6,4 \\ \text { Lohnempfänger } & -27,1 & -29,0 \\ \quad \text { davon deutsche Männer } & -12,1 & \text { KA } \\ \text { deutsche Frauen } & -25,9 & \text { KA } \\ \text { ausl. Arbeiter } & -56,6 & -65,6 \\ \text { ausl. Männer } & -57,6 & \text { KA } \\ \text { ausl. Frauen } & -39,7 & \text { KA } \\ \text { Produktionsbereich } & \text { KA } & -35,9 \\ \text { Verwaltungsbereich } & \text { KA } & -9,5 \\ \text { Auszubildende } & -8,5 & +2,5\end{array}$

Quelle: Arbeitsbericht IV des IFS München, a.a.O., S. 77

Insgesamt folgert das ISF: „Durchweg waren dies die in der Fertigung eingesetzten, direkt produktiven Arbeitskräfte, unter denen wiederum die Ausländer einen bedeutsamen Anteil, teilweise sogar den überwiegenden Anteil am Personalabbau hatten. So waren bei zwei großen Automobilherstellern 60 bzw. 40 v.H. der zwischen 1973 und 1975 ausscheidenden Werksangehörigen Ausländer. Auch bei den kleineren Unternehmen und bei den Zulieferern traf der Personalabbau vor allem die ausländischen Arbeitnehmer." (Ebenda, S. 111) Für den VW-Konzern ermittelte Dom* bois, daß die Entlassungen vor allem die Arbeiter traf, insbesondere solche aus dem Akkordbereich, insbesondere jüngere Arbeiter mit kurzer Werkszugehörigkeit und insbesondere die unteren Lohngruppen. Darunter waren dann die ausländischen Arbeiter die größte Gruppe.

$40 \mathrm{~b}$ Die Zahl der ausländischen Beschäftigten im verarbeitenden Gewerbe verringerte sich zwischen Sept. 74 und Sept. 75 um 13,9\%. Vg1. ANBA 11/76, S. 1163 
Tabelle 6: Abbau verschiedener Beschäftigtengruppen im VW-Konzern in der BRD

$\begin{array}{lrrrrc} & 31.12 .73 & 31.7 .75 & \begin{array}{l}\text { Veränd. } \\ 75 / 73\end{array} & \begin{array}{l}\text { Anteil } \\ \text { a. Abbau }\end{array} & \begin{array}{l}\text { Anteil a.d. } \\ \text { Belegsch. }\end{array} \\ \text { Gresamtbelegschaft } & 125.787 & 94.761 & -24,7 & & \\ \text { Angestellte } & 14.596 & 13.889 & -4,8 & 2,3 & 11,6 \\ \text { Arbeiter } & 109.283 & 78.838 & -27,9 & 98,1 & 86,9 \\ \text { Ausländer } & 19.954 & 6.529 & -67,3 & 43,3 & 15,7 \\ \text { Deutsche Arbeiter } & 89.329 & 72.309 & -19,1 & 54,9 & 71,0\end{array}$

Quelle: Dombois, a.a.O., S. 15 u. 17

Insgesamt kann also angenommen werden, daß in den Industriebereichen mit hoher Ausländerquote und konjunkturempfindlicher Güterproduktion ein Großteil des Personalabbaus über ausländische Arbeitskräfte abgelaufen ist. Zusammen mit den Ausländern bilden die gering qualifizierten Frauen (41), gesundheitlich verbrauchte oder geschädigte Arbeiter/Angestellte sowie un- und dequalifizierte Arbeitsplatzwechsler und Pendler das Entlassungsreservoir in der Krise. Durch die Rationalisierung im Angestelltenbereich im Verarbeitenden Gewerbe setzen sich auch dort langsam ähnliche Selektionskriterien wie im unmittelbaren Produktionsbereich durch (42) - allerdings mit zeitlicher Verschiebung und in anderen Formen der technisch-arbeitsorganisatorischen Rationalisierung. Andere Entlassungsgrinde liegen bei der Disziplinierung von betrieblich-gewerkschaftlich aktiven Belegschaftsmitgliedern vor, hier geht es maßgeblich um die frühzeitige Eliminierung betrieblichen Konfliktpotentials, die Verringerung zuzüglicher sozialpolitischer Kosten.

\section{Kalküle der Ausländerbeschäftigung:}

Auf den ersten Blick erscheint es logisch, nach einer Art „,volkswirtschaftlichem Senioritätsprinzip“ in der Krise als erstes die Ausländerbeschäftigung wieder abzubauen, die in der Hochkonjunkturphase ausgeweitet worden war. Dieses Argument

41 Zur Personalpolitik von VW gegenüber Frauen in der Krise vgl. Claudia Pinl, Arbeitslose Frauen - Schlußlichter der Konjunktur, in: Vorgänge 23, S. 26

42 Vgl. Autorengemeinschaft, Zur Beschäftigungslage der Angestellten, in: Mitteilungen der Arbeitsmarkt; und Berufsforschung, Heft 3/76, S. 302 ff. 
bleibt aber nur logisch, wenn nur die Produktionspotentiale mit Ausländern besetzt wurden, die den Spitzenbedarf der Hochkonjunktur befriedigten und damit keine Umstrukturierung der volkswirtschaftlichen und einzelbetrieblichen Produktion verbunden war. Ein Überblick über die Einsatzbereiche sowie die Arbeits- und Lebensbedingungen der ausländischen Arbeiter in der BRD zeigt aber, daß sie neben der Funktion der Kapazitätsausweitung auch wesentlich eine Rentabilitätsfunktion haben: durch unterdurchschnittliche Löhne, Arbeitsbedingungen, Aufstiegs- und Qualifizierungsanforderungen auf der einen Seite, ein überdurchschnittliches Arbeitsvermögen und einen hohen Mobilitätszwang auf der anderen Seite (43). Die Arbeitskosten pro Produktionsarbeiter werden durch die Ausländerbeschäftigung wesentlich gesenkt. Der Druck auf die Arbeitskosten nimmt in der Krise noch wesentlich zu und würde unter diesem Gesichtspunkt eher eine Ausweitung der Ausländerbeschäftigung nahelegen. Aus der Sicht des Unternehmers hat sich die Stellung der ausländischen Arbeiter in der Krise durchaus verbessert: ihre betrieblich-gewerkschaftliche Position im Betrieb ist so schwach, daß sie sich gegen Lohnabbau und Arbeitsintensivierung kaum wehren können; sie sind als Angelernte im Betrieb leicht umsetzbar; ihr Arbeitsvermögen und ihre Arbeitsbereitschaft ist überdurchschnittlich und sie akzeptieren am ehesten hohe Arbeits- und Gesundheitsbelastungen. In all diesen Punkten sind sie vergleichbaren einheimischen Arbeitskräften überlegen (44). Zur Erklärung der hohen Entlassungsquoten bei Ausländern müssen also noch zusätzliche Gesichtspunkte herangezogen werden:

(1) Die mehrheitliche Beschäftigung in Produktionsabteilungen, die generell am stärksten von Produktionseinschränkungen betroffen werden;

(2) die personalpolitische Strategie, einen Teil der Belegschaft (sogenannte Randbelegschaft) nur vorläufig oder zeitlich beschränkt einzustellen;

(3) die Minimierung der sozialen und politischen Konflikte bei Entlassungen;

(4) die Durchsetzung des Inländerprimats über die staatliche Arbeitsmarktpolitik.

Diese Einzelaspekte können aber hier aus Platzgründen nicht näher ausgeführt werden (45). Gerade aber der letzte Punkt deutet daraufhin, daß der staatlichen Arbeitsund Sozialpolitik bei der Segmentierung des Arbeitsmarktes große Bedeutung zukommt. Dieser Gedanke wäre auch für andere diskriminierte Beschäftigungsgruppen zur Erklärung ihrer betrieblichen Konkurrenzsituation zu konkretisieren.

43 Vgl. Eckart Hildebrandt/Werner Olle, Ihr Kampf ist unser Kampf - Teil I, Offenbach 1975

44 Die Unternehmensbefragung von Bullinger/Huber a.a.O., ergab, daß die besonderen Vorteile der Ausländerbeschäftigung in ihrer Arbeitsbereitschaft unter ungünstigen Arbeitsbedingungen, ihrem Fleiß und ihrer Bereitschaft zu Überstunden gesehen wurden. Nachteihg wurde ihre hohe Fluktuation beurteilt, was aber zu interpretieren ist. Vgl. dort S. $18 \mathrm{ff}$.

45 Vgl. dazu aber E. Hildebrandt, W. Olle, Ihr Kampf ist unser Kampf - Teil II, erscheint demnächst im Verlag 2000, Offebach 1977 


\section{Teil II}

Die neue Strategie des Kapitals: Felxibilisierung des betrieblichen Arbeitsvolumens statt Neueinstellungen

Die Krise seit 1973 hat in den Unternehmen zu einer differenzierteren und längerfristigen Personalpolitik geführt. Die Problemstellung der Personalpolitik hat sich von der Arbeitsbeschaffung zum Personalabbau hin verlagert. Der darin angelegte Lernprozeß beinhaltet, zukünftig noch stärker darauf zu achten, zumindest einen Teil der Belegschaft ohne größere finanzielle und politische Kosten disponibel zu halten. Die Strategie einer disponiblen Belegschaft schließt ein:

1. Die generelle Verringerung der Belegschaften durch technisch-arbeitsorganisatorische Rationalisierung, durch gesteigerte Ausnutzung des Arbeitsvermögens der Beschäftigten und die Abdeckung von Produktionsspitzen durch externe Produktionen/Arbeitskräfte.

2. Die Steigerung der innerbetrieblichen Mobilität der Belegschaft.

3. Abbau der rechtlichen und finanziellen Schranken von Entlassungen, Steigerung der überbetrieblichen Mobilität.

Eine so definierte Strategie geht also über pragmatisches Unternehmerverhalten in einer Phase unsicherer Konjunkturentwicklung weit hinaus.

Die Entwicklung von neuen Instrumenten ist verstärkt notwendig, da einige Mobilitätspotentiale in der nächsten Phase nicht mehr in dem Umfang zur Verfügung stehen:

durch den Anwerbestopp für ausländische Arbeiter und die restriktive Handhabung der Ausländergesetze ist das Potential der Arbeitsimmigranten, die maßgeblich die Beschäftigungsanpassung in dieser Krise getragen haben, verringert und kann in der alten Form nicht erneut aufgebaut werden. Hinzu kommt die Ausweitung der Schutzbestimmungen für besonders bedrohte Arbeitskräftegruppen durch Gesetze (z. B. Ausweitung des Kündigungsschutzes auf unter 18-Jährige seit dem 9.7.76) und Tarifverträge (hier insbesondere Schutz älterer Arbeitskräfte vor Entlassung und Umsetzung).

Elemente einer neuen Strategie, die auf eine langfristige Senkung der Lohnkosten und die Flexibilisierung der Belegschaften hinauslaufen, lassen sich derzeit noch nicht deutlich ausmachen (46). Die Phase seit Mitte 1975 kann durch die rigide Ver-

46 Eine Skizze dieser Strategie bringt Martin Posth, Personalabbau in der Unternehmung, in: Personal, Heft 3/75, S. $114 \mathrm{ff}$ :

"Im Rahmen einer vorausschauenden Personalabbauplanung kommt es darauf an, die Mobilität, Flexibilität und Substitutionalität der betrieblichen Arbeitskräfte so zu steuern, daß jederzeit personelle ,Abbaureserven ${ }^{6}$ in Form von leicht aus der Gesamtbelegschaft herauslösbaren, um die Stammbelegschaft gruppierten, Mitarbeitern zur Verfügung stehen. Das kann beispielsweise erreicht werden durch:

- Verknüpfung von betrieblicher Personal- und Bildungsplanung

- Jahresverträgen mit ausländischen Arbeitnehmern (nicht EG) 
meidung von Neueinstellungen und -- mit starken Unterschieden in den einzelnen Branchen - die weitere, langsame Schrumpfung der Belegschaften durch technischarbeitsorganisatorische Umstellungen und Neuerungen beschrieben werden. Dies zeigen sowohl eine Umfrage der Handelskammern bei den Großbetrieben ihrer Region (47), als auch die Äußerungen der verschiedenen Unternehmensleitungen (48).

Interessant für die Durchsetzung dieser Beschäftigungsstrategie ist, daß wenig Widerstand von den BR entgegengesetzt wird. Das hat zwei Gründe: erstens hat sich das Unternehmerverhalten gegenüber den BR insofern gewandelt, als die BR in die Probleme betrieblicher Antikrisenmaßnahmen einbezogen und an einzelbetriebliche Rentabilitätsüberlegungen gebunden werden. Dabei ist besonders auf die Funktion der Wirtschaftsausschiisse hinzuweisen ( $§ 106 \mathrm{ff}$. BetrVG). Zweitens wollen sie möglichst

- befristete Arbeitsverhältnisse

- Einrichtung von Hausfrauenschichten

- Beschäftigung von Teilzeitkräften

-- Erstellung von Rentnerwerkstätten

- Erteilung von Werksaufträgen an Fremdfirmen

- Beschäftigung von Leiharbeitnehmern/Personal-Leasing

- Flexible Gestaltung des Produktionsprogramms

... Zur vorbeugenden ,Freisetzungsplanung‘ gehört auch eine vorzeitig mit dem Betriebsratvereinbarte, Auswahlrichtlinie' ( $\$ 95$ BetrVG). Gleiche Überlegungen gelten für die Erstellung eines Sozialplans ( $\$ 112$ Betr VG) ... .

Geradezu selbstverständlich sollte heute die rechtzeitige Einbeziehung des Betriebsrates in alle personalplanerischen Maßnahmen unabhängig und oberhalb gesetzlich/tariflicher Zwänge sein. Auf diese Weise ist sichergestellt, daß beide Seiten von vornherein für alle diesbezüglichen Aktivitäten gemeinsam die Verantwortung tragen, was die Durchführung personellen Abbaus wesentlich erleichtern dürfte."

Die Phasen der Veränderung betrieblicher Personalpolitik beschreibt Max Kruk: „Mit hoher Wahrscheinlichkeit kann man voraussagen, daß die Arbeitslosigkeit diesmal weniger rasch zurückgehen wird als nach früheren Schwächeperioden. Zunächst werden die Unternehmen bei wachsenden Aufträgen, wie früher auch, die Kurzarbeit abbauen oder beseitigen. Wegen der inz wischen erreichten Produktivitätssteigerung wird diese Periode relativ lang sein. In einer zweiten Phase werden die Unternehmen dann zu Überstundenarbeit übergehen (was die Belegschaft erfahrungsgemäß gern mitmacht). Und erst relativ spät, wenn eine Vermehrung der Arbeitsplätze nicht mehr vermieden werden kann, wenn sich außerdem erweist, daß die Besserung kein "Strohfeuer" ist, sondern einen dauerhaften konjunkturellen Aufschwung signalisiert, erst dann werden die Firmen an Neueinstellungen größeren Umfangs denken." $\mathrm{FAZ}$ vom 18.8 .75

47 Eine Zusammenstellung dieser Umfragen wurde noch nicht vorgenommen, die Tendenz deutet aber auf einen weiteren Beschäftigungsabbau und auf Neueinstellungen nur in einigen Einzelbetrieben.

48 „Investitionsziele waren", ließ Mercedes-Chef Joachim Zahn die Aktionäre wissen, ,vor allem die Erhöhung der Flexibilität, die Steigerung der Produktivität und eine weitere Verbesserung in der Arbeitsplatzgestaltung. „Und BMW-Chef Eberhard von Kuenheim will in diesem Jahr 260 Mio Mark in die Rationalisierung und Flexibilität stecken. Die geplante Mehrproduktion von 30000 Wagen können die Münchner nur mit Sonderschichten schaffen - doch neue Autobauer sollen kaum eingestellt werden. Zähen Widerstand gegen Neueinstellungen leistet auch VW-Chef Toni Schmücker, der . . . jetzt mit Sonderschichten und ,Verzicht auf die Spitzen in der Nachfrage ' wieder Gewinne anpeilt." Zitiert nach Spiegel 12/76 v. 15.5.76, S. 89 ff. 
eine Wiederholung der Situation vermeiden, Entlassungen mittragen zu müssen

Durch eine Minimierung der Neueinstellungen verringern sie die Konkurrenz der noch Beschäftigten, erreichen mittels Überstunden/Sonderschichten einen Ausgleich für die Einkommenseinbußen in der Krise und verringern das Risiko, wieder in die Schwächeposition wie in der Krise zu geraten. Die betriebsfixierten, kurzfristigen Kalküle der derzeitigen BR-Politik führen folglich dazu, daß sie sich objektiv der Unternehmensstrategie annähert.

\section{Elemente einer veränderten Personalpolitik}

Da der Artikel sich auf die Formen und Funktionen betrieblicher Abbaumaßnahmen beschränkt, muß er auch an dieser Stelle den ökonomischen Zusammenhang, dessen Ausdruck betriebliche Personalpolitik nur sein kann, unausgeführt lassen. So werden die Weiterentwicklungen der Weltarbeitsteilung (Verlagerung in Niedriglohnländer, zunehmende Importkonkurrenz), die steigende Kapitalintensität der industriellen Produktion, die intensive technische und arbeitsorganisatorische Rationalisierung in den kaufmännischen Bereichen, der Konzentration und Zentralisation von Unternehmen sowie der Branchenstrukturwandel in der BRD den entscheidenden Einfluß auf die Zielrichtung der Beschäftigungspolitik und die Bedeutung einzelner Personalpolitiken/Beschäftigungsformen haben (vgl. auch Prokla 19/ 20/21). Im folgenden geht es nur darum, einige Formen der längerfristigen Flexibilisierung der Belegschaften, die für die nächste Phase an Bedeutung gewinnen könnten, kurz zu beschreiben.

1. Aufbau einer Randbelegschaft durch Ausweitung von Werkverträgen, befristeten Arbeitsverträgen und Leiharbeit: Betriebliche Arbeitsverträge auf Zeit sind nur bei Ausbildungsverhältnissen, in den Forschungs- und Entwicklungsabteilungen häufig sowie bei ausländischen Arbeitern die Regel. Bekannt geworden ist dariber hinaus die Einstellungsaktion auf Zeit bei Opel Rüsselsheim für ca. 2000 Beschäftigte im Herbst 1975. Diese Verträge wurden später auf Druck des BR verländert und in Dauerarbeitsverhältnisse umgewandelt.

Über den quantitativen Umfang, die Einsatzbereiche und die formale Organisation von Werkverträgen und sogenannte Leiharbeit, die sich teilweise überschneiden, ist nur wenig bekannt.

Der gewerbsmäßige Verleih von Arbeitskräften war bis April 1967 durch $\$ 37$ Abs. 3 des Gesetzes über Arbeitsvermittlung und Arbeitslosenversicherung (AVAVG) verboten. Dieses generelle Verbot wurde dann aufgrund einer Klage eines Zeit-Arbeit-Unternehmens vom Bundesverfassungsgericht wegen Unvereinbarkeit mit Art. 12 Grundgesetz (freie Arbeitsplatz- und Berufswahl) aufgehoben unter den Annahmen, daß das Arbeitsvermittlungsmonopol des States wegen der geringen Zahl der ZA-Unternehmen nicht eingeschränkt und zusätzliche Arbeitskräfte mobilisiert würden. Am 7.8.1972 wurde durch das Arbeitnehmerüberlas- 
sungsgesetz (AƯG) ein staatlicher Konzessionierungs- und Kontrollrahmen geschaffen. Mit der Aufhebung des Verbots war die Zahl der ZA-Unternehmen schlagartig angewachsen, der Schwerpunkt der Vermittlungstätigkeit verlagerte sich von den kaufmännischen zu den gewerblichen Berufen. Neben den renommierteren Unternehmen der Branche (zusammengeschlossen im Unternehmensverband für Zeit-Arbeit e.V. seit 1969 und im Bundesverband Personal-Leasing seit 1972) vermehrten sich die sogenannten Sklavenhändler, die weitgehend Ausländer ohne Arbeitserlaubnis vermittelten. So erteilte z.B. das Arbeitsamt Berlin einem Verleihunternehmen einen Bußgeldbescheid über 100 000.- DM, weil es in 1078 Fällen ausländische Arbeiter ohne Arbeitserlaubnis beschäftigt hatte (49).

Der DGB hatte sich von Anfang an für ein Verbot der privaten Arbeitnehmerüberlassung ausgesprochen (50). Auf Gewerkschaftstagen waren Verbotsanträge einstimmig beschlossen worden (DGB, IGM, DruPa, BauSteineErden). Die wesentlichen Gegenargumente sind: der Wegfall der Urteilsbegründungen des BVerfGerichtes, die Abwerbung von Stammarbeitern, die faktische Dominanz kurzfristiger Arbeitsverhältnisse auch beim Verleiher, die erhöhte Fluktuation, die Senkung des Qualifikationsniveaus, das Unterlaufen betrieblicher und tariflicher Sozialpolitik, die Beeinträchtigung der betrieblichen Mitbestimmung und gewerkschaftlichen Organisierung, die Möglichkeit des Streikbruchs.

Ziemlich sicher ist, daß die Zahl von geschätzten 60000 legalen Leiharbeitern 1973 inzwischen kaum abgesunken ist und seit 1975 verstärkt angewachsen ist (51). Dies ergab eine Betriebsumfrage des DGB und wird auch an der Entwicklung des größten ZA-Unternehmens ",adia interim" deutlich, das in den ersten Monaten 1976 bis zu $15 \%$ mehr gewerbliche Arbeitskräfte als im entsprechenden Vorjahreszeitraum ausgeliehen hat und mit einer noch verstärkten Nachfrage rechnet (52). Es sind Fälle bekannt, in denen Betriebe während des Belegschaftsabbaus die Zahl der Leiharbeiter erhöht haben; ein Großbetrieb der Automobilzuliefererindustrie entließ im Mai 75 über Aufhebungsverträge 250 Produktionsarbeiterinnen und setzte kurz darauf eine größere Zahl von Leiharbeiterinnen auf diese Dauerarbeitsplätze. Auf bestimmten Arbeitsplätzen werden hintereinander verschiedene Leiharbeiter beschäftigt. Insbesondere bei Auseinandersetzungen um die Durchsetzung von gewerkschaftlichen Forderungen kam es zu Reibungen zwischen Belegschaften und Leiharbeitern bis hin zum Streikbruch durch Leiharbeiter in einem norddeutschen

49 Vgl. Udo Mayer, Der Schutz von Leiharbeitnehmern und das AÜG, in: Arbeit und Recht, Nr. 12/1974, S. 353 ff., hier S. 353

$50 \mathrm{Vgl}$ Stellungnahme des DGB für den Bericht der Bundesregierung an den Deutschen Bundestag über Erfahrungen bei der Anwendung des AÜG, Düsseidorf den 3.4.74 und Kritische Anmerkungen des DGB zu dem Bericht der Bundesregierung an den Deutschen Bundestag vom 9.Juli 1974 über Erfahrungen bei der Anwendung des AÜG (Bundestagsdrucksache 7/2365), Düsseldorf den 25.9.74

51 Werner Then, Präsident des UZA, prognostiziert, daß bis 1980 ca. $5 \%$ aller Arbeitnehmer Zeit-Arbeiter sein werden, die die derzeit bestehende und abzubauende ,Personalreserve" von durchschnittlich $5 \%$ der Belegschaften ersetzen soilen.

(Vgl. Then, Die wirtschaftlichen und personalpolitischen Vorteile der "Zeit-Arbeit", in: Personal, Heft $4 / 72$, S. 148 ff. 
Raffineriebetrieb (53).

Die Spaltung des betrieblichen Interessenvertretungsgefuges ist grundsätzlich durch die kurzfristige Beschäftigung der Leiharbeiter und ihre rechtliche Zuordnung zum ZA-Unternehmen angelegt. Im ZA-Unternehmen kann eine Interessenvertretung faktisch kaum organisiert werden, da die Leiharbeiter bei den entleihenden Betrieben arbeiten, dort sind sie aber tarifvertraglich und betriebsverfassungsrechtlich nicht eingegliedert. So wurde den BR's des entleihenden Betriebes nur ein Recht auf Unterrichtung vor der Einstellung von Leiharbeitern zugesprochen (BAG v. 5.74). Die Spaltungsfunktion hat hier gesetzliche Form angenommen.

2. Ebenso bedeutsam scheint die Strategie zu sein, sich eine externe Belegschaft aufzubauen. Dies geschieht durch die zeitweise Vergabe von Fremdaufträgen und die Abwälzung von Produktionsschwankungen auf Zulieferbetriebe. Diese Strategie ist mit zunehmender

aufzubauen. Dies geschieht durch die zeitweise Vergabe von Fremdaufträgen und die Abwälzung von Produktionsschwankungen auf Zulieferbetriebe. Diese Strategie ist mit zunehmender Unternehmensgröße wirkungsvoller zu verfolgen (54).

3. In gewissem Ausmaß läßt sich auch die Arbeitszeit der Belegschaft flexibilisieren. In der Krise wurde sie durch Kurzarbeit und Werksferien verkürzt, im Aufschwung kann sie durch Überstunden und Sonderschichten gestreckt werden. Dabei geht die Extensivierung mit einer Intensivierung der Arbeit einher.

In der Krise war schon ein nur geringfügiger Abbau der Überstunden beobachtet worden, teilweise sogar die Gleichzeitigkeit von Überstunden und Kurzarbeit im gleichen Betrieb. Ein typisches Beispiel ist die Babcock-Gruppe, die im Geschäftsjahr 1974/75 ihre Belegschaft um 1100 Arbeitskräfte verringerte, gleichzeitig aber 4 Mio Überstunden abforderte, was dem Arbeitsvolumen von ca. 2000 Belegschaftsmitgliedein entspricht. Ihr Vorstandsvorsitzender Ewaldsen konstatiert explizit die Harmonie von schrumpender Belegschaft und hoher Überstundenleistung. „Allein mit Überstunden erreicht man die notwendige Flexibilität im Personaleinsatz, aber auch in der Kostenstruktur." (54 a)

Insgesamt hat die Überstundenleistung pro Arbeitnehmer pro Woche mit der Vertiefung der Krise abgenommen: sie betrug im Juli $1972=3.0$, im Juli $1973=$ 3.0, im Juli $1974=2.5$ und im Juli $1975=1.9$ Wochenstunden. In einigen Zweigen und Betrieben der Verarbeitenden Industrie liegt sie aber wesentlich höher (55).

Die ökonomische Rationalität der Unternehmensstrategie ist unmittelbar einsichtig: sie liegt erstens in der Flexibilisierung der ,zuverlässigen“ Stammbelegschaft

53 Vgl. Beitrag des DGB für den zweiten Bericht der Bundesregierung über Erfahrungen bei der Anwendung des $A \dot{U} G$, Düsseldorf den 12.3.76 sowie weitere Ausführungen dazu vom 29.3.76

54 Unter diesen Punkt gehören auch die derzeitigen Verhandlungen, bei Audi-NSU Mercedes-Kombis zu bauen. Vgl. Handelsblatt v. 3.11.76

54 a Handelsblatt v. 19.2.76

$55 \mathrm{Vg1}$. handelsblatt vom 16.2 .76 
und der Verringerung zukünftiger Betriebskonflikte umKurzarbeit und Entlassungen, zweitens in den kalkulierbar niedrigeren Kosten der Mehrarbeit. Verursacht diese nur einen Mehrarbeitszuschlag von $25 \%$ in der Stunde, so entstehen bei Neueinstellungen: Anwerbungs- und Einstellungskosten, Kosten für Arbeitsplatzinvestition, Einarbeitungskosten; zusätzliche soziale, gesetzliche Lohnneben- und tarifliche Lohnkosten. Für die erste Gruppe der „Fluktuationskosten“" wurden bei einem Facharbeiter schon 26.000,- DM errechnet (56). Bei der zweiten Kostengruppe ergibt sich eine Einsparung von $25 \%$ der Lohnkosten (57). Noch nicht eingeschlossen in die Berechnung sind die Kosten eines zusätzlichen Arbeitsplatzes.

Im Gegensatz zu den Überstunden sind Sonderschichten in der Krise voll eingestellt worden, gewannen aber besonders in der Automobilindusirie schon mit den ersten Auftragsausweitungen wieder an Bedeutung. Teilweise schon einen Monat nach der letzten Kurzarbeitsphase wurden Anfang 1975 wieder die ersten Sonderschichten beantragt.

Überstunden, Sonderschichtenund Einstellungen waren für die Betriebsräte Ansatzpunkte, Forderungen zur Verringerung der Arbeitsplatzbedrohung einzubringen. Die Grundlage bildet die Mitbestimmungspflicht des BR nach \& 87 Abs. 1, Ziff. 3 BetrVG, die außer in akuten Notständen und bei Einzelmehrarbeit gegeben ist. In vielen Fällen wurde durchaus erkannt, daß das Akzeptieren von Mehrarbeit außer der kurzfristigen Einkommenssteigerung eine Reihe von Nachteilen beinhaltet wie: Druchbrechen des Achtstundentages bzw. der Vierzigstundenwoche und damit gleichzeitig Schwächung von zukünftigen Forderungen nach Arbeitszeitverkürzungen, die Uberforderung des Arbeitsvermögens mit gesundheitlichen Folgeschäden und letztlich Entsolidarisierung durch ind ividuelle Einkommensverbesserungen bzw. -differenzierungen innerhalb der Belegschaft und im Verhältnis zu den Arbeitslosen.

Dementsprechend wurden von Belegschaften, VL und BR u. a. gefordert:

- Festlegung einer Zahl von gleichzeitigen Neueinstellungen;

- Verbesserung der betrieblichen Personalplanung;

- Kündigungsverbot für einen bestimmten Zeitraum;

- Erholungspausen zur Kompensation der Mehrbelastung (58)

Exemplarisch sind im folgenden die Auseinandersetzungen um solche Forderungen in zwei Betrieben dargestellt.:

a) Versuch der Durchsetzung einer Beschäftigungsgarantie bei der Firma A. Pierburg Autogerätebau $K G$

1975 waren bei Pierburg 760 Entlassungen geplant. Durch die Einigungsstelle wurde diese Zahl auf 450 herabgesetzt, die bis zum 30.11.75 durch Fluktuation oder Aufhebungsverträge ausscheiden sollten (vgl. Express 6/75, S. 10).

Schon kurz nach der Abfindungsaktion wurde deutlich, daß ca. 270 Arbeitsplätze neu zu

56 Vgl. das Berechnungsbeispiel im Handelsblatt vom 21.12.76, in dem die Einarbeitungskosten mit 23000 DM den weitaus größten Posten ausmachen.

57 Vgl. die genaue Aufstellung bei Ernst Wolf/Gunter Rose, Mehrarbeit bei hoher Arbeitslosigkeit - Herausforderung für Betriebsrat und Gewerkschaft, in: Der Betriebsrat $8 / 76$, S. $298 \mathrm{ff}$.

58 VgL Referentenleitfaden des DGB, Themenkreis Betrieb - Auswirkungen der Krise für die Arbeitnehmer im Betrieb und Möglichkeiten gewerkschaftlicher Gegenwehr - August 1976 , S. $30 \mathrm{f}$. 
besetzen sein würden. Darauf baute der BR eine Strat egie auf, durch einen Arbeitsgerichtsspruch eine Beschäftigungsgarantie zu erhalten. Er verlangte vom Unternehmer eine Beschäftigungsgarantie von einem Jahr, was dieser ablehnte. Pierburg versuchte Neueinstellungen unter Verletzung einer Betriebsvereinbarung vorzunehmen, nach der alle Stellenausschreibungen innerbetrieblich $m$ it genauer Tätigkeitsbeschreibung und in allen Sprachen stattfỉnden müssen. Im daraufhin angestrengten Arbeitsgerichtsprozeß $(\S \S 99,100)$ wurde folgender Vergleich erzielt:

- keine betriebsbedingten Entlassungen bis zum 30.9.76 mit Ausnahme der nach dem 1.10.75 Eingestellten;

- beschäftigte Leiharbeiter werden ersetzt oder in ein ständiges Arbeitsverhältnis überführt; Leiharbeiter werden nur noch in Ausnahmefällen eingestellt:

- Ausschreibungen werden nach der o.g. Betriebsvereinbarung durchgeführt.

b) Betriebsratspolitik bei der Beantragung von Sonderschichten im Aufschwung bei der ADAM OPEL $A G$

Zur Vorgeschichte: 1972 wurden an 26 Samstagen Sonderschichten gefahren, 1973 waren es 21 bis einschließlich Oktober. Im Dez. 73 erfolgte dann plötzlich die erste Kurzarbeitswoche. 1974 gab es über 10 Wochen Kurzarbeit, die der Mehrheit der Betroffenen Lohneinbußen zwischen 200.- und 400.- DM brachten. Zwischen Sept. 73 und Nov. 74 verringerte sich die Belegschaft von Opel Bochum um $25 \%$ (von 19583 auf unter 15 000). Dies geschah maßgeblich durch zwei Aufhebungsaktionen im Juni und Nov.74, über die ca. 3700 Beschäftigte entlassen wurden. Bei Opel/Rüsselsheim verringerte sich die Belegschaft von 38000 auf 27000 . Anfang 1975 gab es im Januar noch 8 Tage Kurzarbeit, im Februar noch 6 und im März plötzlich wieder zwei Sonderschichten. Ebenfalls im März fanden die ersten Neueinstellungen statt. Insgesamt wurden 1975 an Samstagen 30 Sonderschichten gefahren und über 12.000 Neueinstellungen vorgenommen (Erhöhung der Belegschaftsstärke um 8400 gegenüber März 75).

Gegenüber dieser Unternehmenspolitik des „Heuerns und Feuerns" versuchte der BR sehr fruih, d.h. seit den ersten Neueinstellungen und Sonderschichten, die unternehmerische Personalpolitik im Interesse der Arbeitsplatz- und Einkommenssicherung einzuschränken. Dazu gehörten folgende Vereinbarungen:

a) ab Mitte 1975 wurden iiber 1000 Hilfsarbeiter mit nur befristeten Verträgen eingestellt. Hier bewirkte der BR erst eine Verlängerung, dann eine Umwandlung in unbefristete Verträge. Weitere Benachteiligungen der Neueingestell ten lagen in ihrem Qualifikationsverlust, der Eingliederung in die niedrigsten Lohngruppen, der kurzen Anlernung und unzureichenden Unfallbelehrung.

b) Schon frühzeitig konnten bei den Vereinbarungen über Sonderschichten Klauseln abgeschlossen werden, die betriebsbedingte Entlassungen und Kurzarbeit für z. B das nächste halbe Jahr ausschlossen. Diese Klauseln wurden bei den jeweils nächsten Sonderschichten-Verhandlungen verlängert.

c) Darüberhinaus wurde die Errichtung eines Kurzarbeits-Fonds durch das Unternehmen gefordert, über den im Falle erneuter Kurzarbeit die Differenz zwischen Kurzarbeitergeld und Net tolohn ausgeglichen werden soll. Dieses Modell ist in Anlehnung an den IG Chemie-MTV von 1974 entwickelt, nach dem die Differenz zwischen Kurzarbeitergeld und 90\% des Nettolohns vom Unternehmen ausgeglichen wird. Die Unternehmensleitung lehnte ab, ebenso die zum ersten Mal in der Geschichte des Rüsselsheimer Werkes angerufene Einigungsstelle. Die Forderung wurde vom BR-Vorsitzenden Heller bei den nächsten Verhandlungen immer wieder aufgegriffen. Seit Herbst 76 wird sie auch von den BR bei VW und Ford unterstiitzt, ebenso vom IGM-Vorsitzenden Loderer.

d) Seit den BR-Wahlen 1975 wurde bei Opel-Rüsselsheim die Begrenzung/Verringerung der Arbeitsbelastung stärker in den Vordergrund gestellt. Die Durchsetzung der 40-Stunden-Woche (,,Samstag gehört Vati uns") sollte nicht durch ständige Überstunden/Sonderschichten unterlaufen werden, sondern das Unternehmen zu einer „integrierten Personalpolitik" gezwungen, plötzlicher Auftragsdruck zur Durchsetzung längerfristiger Humanisierungsmaßnahmen genutzt werden. So wurde als eine Gegenleistung zur Gewährung von 6 Sonderschichten im Herbst 75 eine 10-minütige Hitzepause bei einer Temperatur von über $30 \mathrm{Grad}$ Celsius erreicht. Einschneidender war die Vereinbarung vom Januar 76, nach der allen Akkord-Arbei- 
ter eine 10-Minuten-Pause/Schicht zugestanden wurde. Diese Regelung wird als Etappe zu einer grundsätzlichen Bestimmung wie im LRTV II Nordbaden-Nord württemberg angesehen (Ėrholungs- und Bedürfniszeit nach § 3.14). (59)

e) Neben dieser qualitativen Forderung wurden bei den einzelnen Verhandlungen um Sonderschichten auch finanzielle Gratifikationen ausgehandelt; z.B. die volle Bezahlung und Überstundenzuschläge für die Vorholschichten für Dez. 75 und eine Erhöhung des Weihnachtsgeldes.

Die hier skizzierte BR-Politk ist in ihrer Reichweite begrenzt. In letzter Zeit deutet sich an, daß sie sich stärker auf Neueinstellungen und eine Beendigung der innerbetrieblichen Umsetzungen ausrichtet. Gleichzeitig wird versucht, die Rüsselsheimer Strategie über das Unternehmen hinauszutragen und innerhalb der Automobilindustrie zu einer einheitlichen, längerfristigen Strategie zu kommen. Dementsprechend forderte die 8. Betriebsrätekonferenz der Adam Opel ÄG:

- eine Konferenz aller BR der Automobilindustrie, um zu einer gemeinsamen Haltung gegenüber Überstunden/Sonderschichten zu kommen;

- daß sich die IGM für eine Verkürzung der täglichen Arbeitszeit einsetzen solle:

- die Verallgemeinerung der Forderung nach betrieblichen Kurzarbeitsfonds (60).

Diese Beispiele stehen für eine aktive, beschäftigungssicherungsorientierte $\mathrm{BR}$ Politik, daneben gibt es die Mehrzahl von Fällen, in denen mögliche Neueinstellunbesserungen ausgehandelt wurden und auch Fälle, in denen mögliche Neueinstellungen vom BR zugunsten von Arbeitsintensivierung und Einkommenssteigerung für die reduzierte Belegschaft verhindert wurden. Ein Beispiel dafür scheint die Erhöhung der Akkord-Verrechnungsgrenze im Werk Sindelfingen von Daimler-Benz im Herbst $1976 \mathrm{zu}$ sein. Um die Produktion um $3 \%$ zu erhöhen, bot die Unternehmensleitung dem BR an, entweder 400 neue Arbeitsplätze zu schaffen oder die Akkorde anzuheben und damit den Jahreslohn der Produktionsarbeiter um ca. 700 DM zu erhöhen (61). Da es kurz zuvor im Werk einen Lohnstreik gegeben hatte, êntschied sich der $\mathrm{BR}$, ohne die Belegschaft über die andere Alternative zu informieren, für Lohnerhöhung und Intensivierung. Er konnte damit den Erfolg für sich buchen, die Belegschaftsforderung nachträglich erhandelt zu haben und gleichzeitig das zukünftige Risiko von Entlassungen verringert zu haben. Es kann aber angenommen werden, daß sich die Belegschaft bei offener Diskussion aus zwei einfachen Gründen für Neueinstellungen entschieden hätte: erstens ist es nicht einsichtig, warum die jetzt Arbeitslosen nicht im Betrieb mitarbeiten sollen, wo sie doch als Arbeitslose von den noch Beschäftig. ten bezahlt werden. Zweitens verringert sich durch Neueinstellungen die eigene Arbeitsbelastung. Etliche Streiks für Neueinstellungen (62) bzw. die Wiedereinstellung kïrzlich Entlassener bestätigen, daß eine solche Politik teilweise von den Belegschaften getragen werden kann.

59 Die derzeitige Hauptforderung strebt an eine effektive Arbeitszeitverkäurzung auf $71 / 2$ Stunden bei Schichtzeiten von 6-14 und $14-22$ Uhr mit bezahlter Pause (vgl. Express $1 / 76$, S. 4)

60 Vgl. Frankfurter Rundschau vom 25.9. und 31.10.76

61 Vgl. Informationen der Plakat-Gruppe/Stuttgart vom 7.10.76

62 So im Madsack-Konzern in Hannover und der Aeroquip GmbH in Hannover 


\section{Teil III}

Bisherige Erfahrungen aus der Betriebspolitik in der Krise als Ansatzpunk te einer Gegenstrategie

Zwischen der Kennzeichnung der derzeitigen Krise als längster und schwerster wirtschaftlicher Krise der BRD seit 1949 und dem sozialen und politischen Konfliktpotential als Folge verschlechterter Arbeits- und Reproduktionsbedingungen besteht eine Differenz, die erklärt werden muß. Die Benennung der Mechanismen, über die teilweise erhebliche materielle Verschlechterungen relativ konfliktlos durchgeführt werden können, soll hier nur als erste Schlußfolgerungen aus dem vorgelegten Material erfolgen. Inwieweit damit bereits Grundzüge einer Gegenstrategie gegeben sind, muß vorerst offenbleiben; diese Diskussion soll aber in der Prokla weiterhin geführt werden.

1) Die Krise seit $1973 / 74$ folgte auf eine lange Phase relativ stabilen Wachstums, mit dem sich das Selbstverständnis der BR und auch der verschiedenen Beschäftigtengruppen indentifizierte. Gewerkschaftliche Tarifpolitik und Belegschaftforderungen bewegten sich auf dem geraden Pfad kontinuierlicher, aber beschränkter Einkommenszuwächs, Verkürzung der Arbeitszeit, Verbesserung der Arbeitsumgebung und rechtlicher Absicherung des sozialen Status in und außerhalb des Betriebes. Gerade die starke Identifikation mit dem Wideraufbau nach 1945 steht der Einsicht entgegen, daß der selbstgeschaffene Wohlstand aus der Hand genommen werden könnte, daß man „wieder“" hilfsloses Objekt eines krisengeschüttelten Kapitalismus werden könnte, den man nach 1945 entgültig überwunden oder zumindest modifiziert zu haben glaubte. Auf der anderen Seite scheint doch noch eine latente Angst vor Zuśtänden wie in der Weltwirtschaftskrise verbreitet zu sein, die zur Verdrängung von Krisenerscheinungen und zur Hoffnung auf den Aufschwung führt.

Nur über solchen Erfahrungshintergrund und Bewußtseinsstrukturen erscheint erklärlich, daß auch bei den durch Arbeitslosigkeit am stärksten gefährdeten Gruppen kein aktives Verhalten gegen Arbeitslosigkeit vorzufinden ist. Das Vertrauen in die ,Wirtschaftskraft" läßt die Gefährdeten abwarten, bis es zu spät ist und sie von den noch Beschäftigten als Versager ausschließt. Dann fehlen den Betroffenen auch die notwendigen Informationen (z. B. über das AFG, über arbeitsgerichtliche Möglichkeiten) und die Möglichkeiten der Kollektivierung ihrer sozialen Anstrengungen gegen die Verschlechterung ihrer Lebenslage. Gleiches gilt für die betrieblichen Möglichkeiten der Gegenwehr. Es gibt kaum Beispiele dafür, daß sich Belegschaften außer durch noch intensivere Arbeitsverausgabung auf absehbaren Belegschaftsabbau vorbereitet hätten, Betriebsräte zu einem bestimmten Verhalten gezwungen hätten etc.

Betriebliche und gewerkschaftliche Interessenvertreter befanden sich in einer ähnlichen Lage. Ihr Informationsstand über ökonomische Entwicklungstendenzen, über betriebliche Entlassungsstrategien, über Erfahrungen mit Abwehrstrategien gegen Belegschaftsabbau aus anderen Betrieben, Branchen oder sogar Ländern, schließlich über. ihre eigenen Möglichkeiten nach oder knapp neben dem BetrVG waren gering. Diese Informationen wurden in jedem Betrieb erst sukzessive mit dem Belegschaftsabbau im eigenen Betrieb gesammelt, und entsprechende eigene Erfahrungen 
nachgeholt. Für viele BR war die Krise nicht die extensive Anwendung eines Abwehrinstrumentariums, sondern ein eingeschränkter Lernprozeß über dessen Wirkungsweise. Somit war der materielle Erfolg der Gegenwehr auch gering.

Mit diesen Aussagen ist die Frage nach den Möglichkeiten gestellt, betriebliche Erfahrungen kollektiv zu verarbeiten, zu verallgemeinern und aufzubewahren, also nicht als Einzelbetriebs-BR pragmatisch den konjunkturellen Entwicklungen und den Veränderungen des Rechtsrahmens hinterherzuhetzen. Das wäre wohl nur über eine Ausweitung und Konzeptionsänderung der gewerkschaftlichen Funktionärsschulungsund Bildungsarbeit

Ausweitung und Konzeptionsänderung der gewerkschaftlichen Funktionärsschulungsund Bildungsarbeit möglich. Darin läge auch ein Ansatzpunkt für eine Gegenstrategie zu Entlassungen, indem betriebliche Unterauslastung zu Lehrgängen über ökonomische Krisenprozesse, Auswirkungen und Gegenstrategien genutzt werden können.

2) Die Verallgemeinerung von Erfahrungen und Informationen fällt insbesondere dann schwer, wenn die Kompetenz der Krisenabwehr institutionell auf den BR fixiert ist. Bis auf die Aktionen von Belegschaften gegen Betriebsänderungen, insbesondere Stillegungen, beschränken sich die Abwehraktionen auf Interventionen der Betriebsräte. Die hoffnungsvoll angelegte Vertrauensleutestrategie wäre wohl in der Lage gewesen, die Einbindung der BR-Arbeit in den Erfahrungs- und Lernprozeß der Belegschaftèn langfristig herzustellen und ihn damit auch aus den Bìndungen an die Unternehmensleitung und aus der Beschränkung auf die Anwendung rechtlich normierter Interventionen abzulösen.

3) Durch die Verrechtlichung der BR-Position über das BetrVG und seine relative Isolation von der Machtposition der Gesamtbelegschaft verlagert sich die BR-Arbeit auf die Durchsetzung staatlicher Normen gegenüber der Unternehmensleitung und teilweise auch gegenüber der Belegschaft. Diese Normen ștehen ihm aber auch selbst als Parlamentsgesetze fremd gegenüber, sind nicht Resultate gewerkschaftlich orientierter Betriebsratsarbeit. Sie verweisen ihn zudem letztlich wieder auf das Unternehmerinteresse oder auf gesellschaftliche Instanzen wie Arbeitsgericht und Einigungsstelle.

Es kann nicht angezweifelt werden, daß der gegenwärtige Stand von Arbeitsgesetzen ein im internationalen Vergleich hohes Niveau an Mitbestimmung und sozialer Sicherheit auch dann garantiert, wenn eine Gegenwehr von den Betroffenen nicht stattfindet, sondern diese nur ihre Rechte wahrnehmen. Die Kehrseite ist aber, daß die Form und das Niveau der gesetzlichen Absicherung nicht als Obergrenze des Erreichbaren interpretiert und auch nicht als Einengung der Widerstandsformen akzeptiert werden darf. Genau das passiert aber, die Einhaltung der Gesetze wird als optimales Ergebnis von BR-Politikgesehen, die Beteiligung der Belegschaft möglichst sogar verhindert, weil sie in diesem Rechtsrahmen nicht vorgesehen und integriert ist.

Zudem mußten die BR's die Erfahrung machen, daß die Wirkung der vielen Schutzgesetze weniger in einer Verringerung der Krisenlasten besteht, sondern eher in einer Verzögerung und Umverteilung. Verzögerung meint, daß Kündigungen durchgesetzt werden können, daß die finanziellen Folgen aufgeschoben werden. Umver- 
teilung meint, daß Schutzbestimmungen für die eine Gruppe kurzfristig für eine andere soziale Gruppe das Arbeitsplatzrisiko erhöhen, daß längerfristig die Unternehmen auch die Arbeitskräfte loswerden, die ihnen nicht produktiv genug sind. $\mathrm{Zu}$ sammen mit dieser Desillusionierung gerieten die BR's in den Zwang, sich in den vorgegebenen rechtlichen Formen zu bewegen und das heißt, die von ihnen höchstens modifizierte Unternehmenspoltik mitzutragen. Der Preis der Ausnutzung des vorgegebenen Handlungsspielraums ist die Vertretung der Ergebnisse gegenüber der Belegschaft. Da BR-Politik derzeit Unternehmenspolitik in den seltensten Fällen entscheidend für die Beschäftigten verändert, gerät der BR als Interessenvertreter der Belegschaft in die widerspruchsvolle Situation, der Belegschaft abgemilderte Unternehmenspolitik andienen zu müssen.

Es geht hier nicht um die Ablehnung von Kompromißpolitik, sondern um die Einbindung des BR in Verhandlungs- und Vertretungsformen (63), die ihn notwendig von der Belegschaft ablösen und ihm sogar Unternehmerfunktionen zuweisen (z. B. bei der Ablehnung von Entlassungen einen Gegenvorschlag zu machen). Bei der Selektion von zu Entlassenden wird z. B. deutlich, wie inkompetent ein BR für solche Entscheidungen ist, wie er häufig die Diskriminierung von Frauen, Kranken, Jugendlichen und Ausländern verstärkt.

Im Verhältnis zwischen Unternehmensleitung und Betriebsräten hat sich im Rahmen der veränderten Unternehmenspolitik in der Krise eine neue Konstellation ergeben: der BR wird bewußt und teilweise über den vorgeschriebenen Rahmen hinaus in die Entscheidungen zur Rentabilitätssteigerung durch Lohnkostenabbau einbezogen (allerdings nur in diesen Bereich der Unternehmenspolitik!), zum mitverantwortlichen Träger gemacht und noch stärker von den Belegschaften getrennt. Eine wichtige Funktion hat dabei die Schweigepflicht, die die ständige Rückkoppelung mit der Belegschaft verhindert und die Trennung verstärkt.

4) Damit ist auch die Frage gestellt, welche wirtschafts- und sozialpolitischen Alternativvorstellungen ein BR bei der Auseinandersetzung mit der Unternehmenspolitik überhaupt entgegensetzen kann. Im Einzelfall von Kurzarbeit, Entlassungen oder Stillegungen wird sowohl von Gewerkschaftsseite wie von den BR normalerweise auf der Linie argumentiert, daß die Notwendigkeit des Belegschaftsabbaus allein durch eine falsche Unternehmenspolitik entstanden sei, ihre Gegenvorstellungen enthalten stets die quantitative Reduktion des Abbaus. Daraus wird zweierlei deutlich:

Erstens, daß sie trotz dieser personell vereinseitigten Krisenanalyse nicht in der Lage sind, die Ursache zu beseitigen. Sie haben keinen Einfluß auf die Besetzung des Unternehmensmanagements und auf die von ihm durchgeführte Unternehmenspoli-

63 Diese Einbindung zeigt sich deutlich in der Hilflosigkeit, mit der die BR der Entlassungsform der Aufhebungsverträge mit Abfindung gegenüberstehen. Eine Form, die die Unternehmen gerade zur Umgehung der rechtlichen Schutznormen herausgebildet haben, auf die sich BR-Politik zu stiitzen sucht.

In den Grundlinien der Gewerkschaftspolitik zeigt sich die Verkehrung, daß die Erhöhung der Mobilität der Beschäftigten zum gewerkschaftlichen Ziel erklärt worden ist. Das bleibt solange ein Anachronismus, wie keine volkswirtschaftliche, von den Gewerkschaften dominierte Beschäftigungs- und Bildungsplanung existiert. 
tik. Sie können nur im Rahmen der ihnen zugewiesenen Kompetenzen abmildern und modifizieren. Kurz, es gibt keine rechtlichen Möglichkeiten, Entlassungen überhaupt zu verhindern. Die Gegenstrategie der quantitativen Reduktion kann überdies von den Unternehmen in der Weise unterlaufen werden, daß Kurzarbeitsperioden und Entlassungszahlen beantragt werden, in die bereits ein Verhandlungsabschlag einkalkuliert ist, sodaß sich nach der Einigung mit dem BR oder dem Spruch der Einigungstelle in etwa die angestreben Dimensionen ergeben. Dies ist solange möglich, wie die BR die wirtschaftlichen Grunddaten des Unternehmens vorenthalten bleiben und sie die Produktionsstrategie nicht entscheidend mitbestimmen können.

Zweitens, und das erscheint mir wichtiger, orientiert sich die gewerkschaftliche Alternativvorstellung zur Unternehmenspolitik an einem Modell eines sozialen, gesamtwirtschaftlich ausgerichteten und kontrollierten Kapitalismus. Dabei entsteht der Widerspruch, daß sich die gewerkschaftlichen Vorschläge und Initiativen nur auf die Planungs-, Kontroll- und Entscheidungsformen beschränken, während die eigentlichen Mechanismen der Profitproduktion außerhalb des Blickfeldes bleiben. Daraus folgt notwendig, daß selbst bei der Institutionalisierung dieser Planungs- und Mitbestimmungsgremien im Sinne der Profitabilität entschieden werden muß, die dann ; wieder genau die materiellen Wirkungen hervorruft, die die Gewerkschaften und BR mit diesen Gremien beseitigen wollten. Diese These könnte am Beispiel der Gewerkschafts-Argumentation zur VW-Sanierung ausgefuihrt werden.

Die Orientierung auf einzelbetriebliche und volkswirtschaftliche Rentabilitätsund Wachstumsparameter hat dazu geführt, daß eine davon autonome Argumentation der Reproduktionssicherung und -verbesserung nicht mehr existiert. Die Gewerkschaften vergeben sich tendenziell die eigene Position: die Forderungen nach Einkommen und Arbeitsbedingungen primär von den Arbeits- und Reproduktionsinteressen ihrer Mitglieder her zu bestimmen.

5) Das Verhältnis von Unternehmensstrategie und gewerkschaftlicher Gegenkonszeption ist auch in einem in der Krise entscheidenden Bereich unklar: den technisch-arbeitsorganisatorischen Rationalisierungen. Die Euphorie über die Haupttechniken der sog. Humanisierung der Arbeit in ihrer Wirkung auf die Beschäftigten dürfte inzwischen einer nüchterneren Einschätzung gewichen sein. So müssen als Haupteffekte der Humanisierungs-Umstellungen $\mathrm{z}$. B. in der deutschen Automobilindustrie die Flexibilisierung der Produktion, die Steigerung dr Produktivität und die Verringerung des Personaleinsatzes registriert werden. Die punkutelle Verringerung der Arbeitsbelastung und Verbesserung der Arbeitsbedingungen durch die stärkere Entkoppelung der Einzelverrichtungen und durch die Anreicherung des Einzelarbeitsplatzes mit mehr Arbeitsgängen erscheint mehr als Folgewirkung, deren realer Effekt langfristig noch nicht beurteilt werden kann. Die eigentliche gewerkschaftliche Gegenstrategie zur Rationalisierung dürfte eher in der Belastungsbegrenzung und im Belastungsabbau ibber Tarifvertrag liegen (vgl. LRTV II in Nordbaden-Nordwürttemberg). In jedem Fall fehlt der vereinzelten Kritik an der kapitalistischen Rationalisierung und am Produktivitätsziel die Substanz einer Gegenstrategie, ein umfassender und differenzierter gewerkschaftlicher Rationalisierungsbegriff, der dann auch die $\mathrm{Be}$ - 
triebsräte handlungsfähig machen könnte.

Vielmehr sind sie zur Zeit gezwungen, der Rationalisierung zur Verbesserung der Unternehmensrentabilität zuzustimmen und nur auf die sozialen Folgen im Betrieb modifizierend einwirken zu können.

6) Insgesamt zeigt sich, daß die BR aufgrund des Fehlens einer verallgemeinerten gewerkschaftlichen Abwehrstrategie in der Gegenwehr auf sich allein und auf ihren Betrieb beschränkt bleiben. Selbst zu den häufigen und für die BR ziemlich wichtigen Entscheidungssituationen wie Stellungsnahme zu Entlassungen/Kurzarbeit/ Sonderschichten/Überstunden hat es keine überbetriebliche, gewerkschaftlich organisierte Diskussion und Empfehlung gegeben (in den Stellungsnahmen zur Bewilligung von Überstunden im Frühjahr 1976 wurde zwar generell zum Abbau geraten, aber gleichzeitig die Ambivalenz zwischen Verteilung der Arbeit und Aufholen der Lohneinbußen betont). Auf der Tagung der IGM zu „Krise und Reform der Industriegesellschaft" wurde die Diskrepanz zwischen weltwirtschaftspolitischen Modelllösungen der Krise und den Problemstellungen der alltäglichen BR-Arbeit besonders deutlich. Auch die Aufforderung der Opel-BR an die IGM, zu diesen Fragen eine Tagung aller BR der Automobilindustrie zu veranstalten, deutet auf dieses Defizit hin.

7) Besonders in Großunternehmen mit mehreren Zweigwerken zeigen sich die Nachteile der Kompetenzbeschränkung des BR auf seinen Einzelbetrieb. Die Zusammenfassung in Konzernbetriebsräten scheint diese Beschränkung eher auszudrücken als aufzuheben. Mir ist kein Fall bekannt, indem bei einer Betriebsänderung der Konzernbetriebsrat für den betroffenen Einzelbetrieb die Abwehr oder zumindest die Verteilung des Abbaus erwirkt hätte. Der BR ist in erster Linie Vertreter ,seines" Betriebes und damit Konkurrent gegenüber den anderen Unternehmensbetrieben und damit auch gegenüber den anderen BR. Diese Konkurrenz wiederum wirkt dahin, daß er versuchen wird, den Beschäftigungsabbau auf andere Betriebe abzuwälzen, seien es andere Unternehmensteile oder Zuliefererbetriebe (vgl. hier besonders die Strategie der Stornierung von Fremdaufträgen). Die andere Seite des Abwälzens besteht darin, daß der BR daran mitwirken muß, die Produktivität und Rentabilität seines Betriebes gegenüber den anderen Unternehmensteilen zu steigern, um in den Augen der Unternehmensleitung diesen Betrieb unentbehrlich zu machen. Dieser Prozeß läßt sich eindrucksvoll am Kampf der Steinkohlenzechen ums Überleben in den fünfziger und sechziger Jahren belegen; aber auch aus dieser Krise gibt es illustre Beispiele wie Kalldorf/DEMAG. Auch der Konzern-BR im VW-Konzern hatte keine abschwächende und vereinheitlichende Funktion (64).

Das Fehlen einer überbetrieblichen Perspektive der BR-Arbeit und die Beschränkung der Einflußmöglichkeiten auf die Unternehmenspolitik bringt in der Krise noch eine weitere, erstaunliche Wirkung hervor. Ein Teil der BR versucht von sich aus, die Absatzlage des Unternehmens zu verbessern, um Arbeitsplätze im Betrieb zu erhal-

Vgl. Guilden/Peter, a.a.O., S. 44 
ten. Einige versuchten, weitere Aufträge hereinzuholen, die meisten wandten sich an die Kommunen und die Bundesregierung, um Veränderungen der Marktkonstellation zu erreichen. Ältere Beispiele sind die Durchsetzung von erhöhten Absatzgarantien für Steinkohle und die Verschärfung von Importrestriktionen in der Textilindustrie. Widersprechen diese Initiativen schon den strukturpolitischen Vorstellungen des DGB, so häufen sich in letzter Zeit die Beispiele, in denen die BR eindeutig gegen politische und soziale Maxime der Gewerkschaftspolitik verstoßen. Besonders häufig ist dies beim Konflikt zwischen Umweltschutz und Erhaltung/Ausbau/Errichtung einer besonders umweltgefährdenden Produktionsstätte.

An diesen Beispielen wird auch deutlich, in welchem Maße die Dimensionen der BR-Politik von der Größe, Marktstellung und volkswirtschaftlichen Bedeutung „ihres“ Betriebes abhängen. Die überwiegende Mehrzahl der Betriebsräte kann sich noch nicht einmal der Illusion hingeben, von ihrer Position aus gegen übergreifende Rationalisierungs- und Umstrukturierungsprozesse angehen zu können. Damit ist ein neues Moment der Spaltung zwischen den Großbetrieben und den unbedeuteren Betrieben angelegt.

8) Ein besonders wichtiges Problem fur die BR-Arbeit scheint mir in der schon angedeu teten Abwälzung der Krisenauswirkungen zu liegen. Der Versuch der Abwälzung auf andere Betriebe wurde bereits benannt. Eine noch schwerwiegendere Abwälzung findet aber dadurch statt (hier wird ja das Problem nur verlagert), daß die derzeitige BR-Politik dazu beiträgt, die Lasten auf bestimmte Bevölkerungsgruppen wie Frauen, Jugendliche, Gesundheitsschwache und Ausländer zu konzentrieren. Das wird bewirkt einmal durch das Intrument des Entlassungsstopps, mit dem die Arbeitskräfte, die neu in den Arbeitsmarkt eintreten oder woanders entlassen wurden, keine Möglichkeit zur Neueinstellung haben. Für diese besteht auch keine Möglichkeit, in einen Kontakt zur Belegschaften und Gewerkschaften als Vorform einer Arbeitsbeziehung zu kommen bzw. diesen Kontakt zu halten. Das liegt zum anderen an den Selektionskriterien bei Belegschaftsabbau, die sich in der Auseinandersetzung zwischen Unternehmen, Belegschaften und BR herausgebildet haben. Die Anwendung von Kriterien wie Leistungsfähigkeit, Dauer der Betriebszugehörigkeit, Geschlecht und Nationalität verlängert und verstärkt genau die gesellschaftlichen Diskriminierungen, gegen die die Gewerkschaften programmatisch angetreten sind. Die faktischen Ergebnisse der Entlassungspolitik lassen die Ansätze zur Integration der Frauen in die Erwerbstätigkeit, die Qualifizierung und Verstetigung der Beschäftigung gering Qualifizierter, die Verbesserung der Arbeits- und Lebensbedingungen gesundheitlich Verschlissener, die Integration der Arbeitsimmigranten hilflos erscheinen. Das zynische Extrem stellt die Frühentlassung der ausländischen Arbeiter dar mit der Begrïndung, daß sie ja in ihrem Heimatland Arbeit finden könnten und sie die Entlassung sozial nicht so schwer treffe.

Diese Politik trägt auch dazu bei, die sozialen Probleme der Krise geringer erscheinen zu lassen, als sie in Wirklichkeit sind. Damit verringert sie auch den gesellschaftlichen Druck in Richtung auf Gegenstrategien, die allein die Gewerkschaften und BR bei ihren Abwehrmaßnahmen unterstützen könnte. 
Neben den materiellen Folgen für diese Randgruppen und der objektiv spaltenden Auswirkungen dieser „Ausgrenzung“ ist damit eine Schwächung der Gewerkschaften selbst verbunden. Ihre Vertretungs- und Integrationsmacht schränkt sich zunehmend auf die Beschäftigtengruppen und Branchen/Unternehmen ein, die relativ geringfügig von der Krise betroffen sind.

9) In die gleiche Richtung wirkt auch die Dezentralisierung und Delegation der Entscheidungen über das Arbeitsschicksal und das Reproduktionsniveau der Beschäftigten. Unternehmensleitung, Betriebsrat, Arbeitsgericht, Einigungsstelle, Arbeitsverwaltung und Soziallbetreuung zerstuickeln den Gesamtzusammenhang des Entlassungsprozesses, zerren ihn instiutionell und zeitlich auseinander, sodaß der Uberlick und die Kompetenz für die Betroffenen - Voraussetzungen, um die Bewältigung ihrer Probleme aktiv vorzunehmen - zerstört werden. Sie verfügen über keine Instanz, die in der Lage wäre, den Gesamtzusammenhang herzustellen und bearbeitbar zu machen. Die bekannten Folgen sind Passivität und Individualisierung.

Ein wichtiges Moment in diesem Prozeß ist die Verstaatlichung der Interessenwahrnehmung. Sie bewirkt die Trennung von Verursachung (das Unternehmen entläßt) und Interessenwahrnehmungsinstanz (Gesetzgebung, Arbeitsgerichte, Arbeitsverwaltung). Ein Angriff gegen die Maximen des Privatkapitals kann damit nur beschränkt über die quasi-neutrale, gesamtgesellschaftlich strukturierte Sozialstaatsinstanz geführt werden. Beschränkt heißst, daß der Staat nur im Rahmen seiner Fürsorgepflicht bezüglich der Milderung der sozialen Folgen in Anspruch genommen werden kann.

10) Umgekehrt formuliert: Der Überblick über die verschiedenenEntlassungsvorgänge zeigt doch, daß Widerstand dann möglich war, wenn die Entlassungen auch als solche in größerer Zahl gleichzeitig auftraten (vergl. Erwitte, Speyer etc.). Dann wurde es doch möglich, daß die Gesamtbelegschaft mobilisiert wurde, die Bevölkerung sich am Protest beteiligte, Verwaltungen und Verbände Stellung nehmen mußten, der Stand der Verhandlungen über die Rücknahme der Entlassungen oder Sozialpläne, über die Weiterbeschäftigung der Entlassenen zum Gegenstand öffentlicher Diskussion und damit auch öffentlichen Drucks wurden. Der Gesamtzusammenhang des Arbeiterschicksals in der Krise wurde präsent und es entstand eine breitere, solidarische Interessengemeinschaft gegen die Abbaumaßnahmen.

Es soll hier nicht behauptet werden, daß erstens in diesen Fällen generell bessere Ergebnisse durchgesetzt werden können, als auf den oben beschriebenen, institutionalisierten Verhandlungswegen (obwohl das teilweise der Fall war), und daßs zweitens den Unternehmen eine andere als die Rationalität des Kapitals aufgezwungen werden könnte. Aber zwei Gesichtspunkte sollten doch stärker hervorgehoben werden: erstens, daß die Kompromißlinie zwischen Unternehmen und Belegschaft bei einer konzentrierten, öffentlichen und gemeinschaftlichen Austragung des Konflikts zugunsten der Belegschaften verschoben werden kann. Zweitens, daß die Lernprozesse für die Beteiligten je nach der Art der Austragung des Konflikts sehr unterschiedlich sein werden. Die derzeitige BR- und Gewerkschaftspolitik fördern 